\title{
Review
}

\section{Nano- and Micropatterning on Optical Fibers by Bottom-Up Approach: The Importance of Being Ordered}

\author{
Marco Pisco ${ }^{1, *(D)}$ and Francesco Galeotti ${ }^{2, *(D)}$ \\ 1 Optoelectronics Group, Department of Engineering, University of Sannio, c.so Garibaledi 107, \\ 82100 Benevento, Italy \\ 2 Istituto di Scienze e Tecnologie Chimiche "G. Natta" (SCITEC), Consiglio Nazionale delle Ricerche, \\ via A. Corti 12, 20133 Milano, Italy \\ * Correspondence: pisco@unisannio.it (M.P.); galeotti@scitec.cnr.it (F.G.)
}

check for updates

Citation: Pisco, M.; Galeotti, F. Nanoand Micropatterning on Optical Fibers by Bottom-Up Approach: The Importance of Being Ordered. Appl. Sci. 2021, 11, 3254. https://doi.org/ 10.3390/app11073254

Academic Editor:

Alessandro Belardini

Received: 6 March 2021

Accepted: 27 March 2021

Published: 5 April 2021

Publisher's Note: MDPI stays neutral with regard to jurisdictional claims in published maps and institutional affiliations.

Copyright: (c) 2021 by the authors. Licensee MDPI, Basel, Switzerland. This article is an open access article distributed under the terms and conditions of the Creative Commons Attribution (CC BY) license (https:// creativecommons.org/licenses/by/ $4.0 /)$.
Abstract: The realization of advanced optical fiber probes demands the integration of materials and structures on optical fibers with micro- and nanoscale definition. Although researchers often choose complex nanofabrication tools to implement their designs, the migration from proof-of-principle devices to mass production lab-on-fiber devices requires the development of sustainable and reliable technology for cost-effective production. To make it possible, continuous efforts are devoted to applying bottom-up nanofabrication based on self-assembly to decorate the optical fiber with highly ordered photonic structures. The main challenges still pertain to "order" attainment and the limited number of implementable geometries. In this review, we try to shed light on the importance of self-assembled ordered patterns for lab-on-fiber technology. After a brief presentation of the light manipulation possibilities concerned with ordered structures, and of the new prospects offered by aperiodically ordered structures, we briefly recall how the bottom-up approach can be applied to create ordered patterns on the optical fiber. Then, we present un-attempted methodologies, which can enlarge the set of achievable structures, and can potentially improve the yielding rate in finely ordered self-assembled optical fiber probes by eliminating undesired defects and increasing the order by post-processing treatments. Finally, we discuss the available tools to quantify the degree of order in the obtained photonic structures, by suggesting the use of key performance figures of merit in order to systematically evaluate to what extent the pattern is really "ordered". We hope such a collection of articles and discussion herein could inspire new directions and hint at best practices to fully exploit the benefits inherent to self-organization phenomena leading to ordered systems.

Keywords: self-assembly; nanosphere lithography; lab-on-fiber; optical fiber sensors; photonic crystals; nanofabrication; nanopatterning; honeycomb structure; periodic nanostructures; biosensors

\section{Introduction}

Nature is both chaotic and ordered. Despite the usual and probably misleading association between entropy and disordered systems [1], in nature, we can find many examples of ordered patterns. The structure of a beehive, a spider's web, the stripes on a zebra's mantle, the ice crystals in a snowflake are just a few cases of perfect periodic repetitions among many others. Sometimes the aim of natural periodic patterns is to create specific color effects by light interference, leading to a diverse array of functions as mate attraction, UV protection, camouflage and sensory enhancement. Similarly, artificial ordered patterns at the nano- and microscale find large applications as photonic elements in different technological fields, and specifically, they play a central role in optical fiber (OF) technology. When coupled to photonic structures, in fact, the OF becomes a technological platform where the waveguiding propagation of light through the fiber is coupled with the light-matter interaction occurring on the photonic element. Consequently, a vast number of sensors and sensing probes based on OFs have been developed in the last decades, contributing to the vision of the lab-on-fiber concept $[2,3]$. To make it possible, the effort 
of researchers is focused on one side to the development of new fabrication approaches able to control precisely the periodic structure at the nanoscale, and on the other side to adapt to the OF the already robust technologies usually applied on silicon wafers or, more in general, on planar surfaces.

Two main approaches-top-down and bottom-up—can be followed to fabricate photonic nanostructures on the OF. The top-down approach is a subtractive process in which the nanostructures are produced by removing material, is generally the most expensive and time-consuming, but also the most precise manufacturing option. It typically produces highly ordered patterns by using robust technologies such as electron-beam lithography, focused ion beam, interference lithography, femtosecond laser writing and two-photon lithography, which have demonstrated its capability to implement complex designs and architectures. The bottom-up methods are additive processes normally based on selfassembly, in which the material elements are used to build up the desired objects. The self-assembly of nano-, micro- or macroscopic objects involves weak local interactions that can include van der Waals, electrostatic and hydrophilic/hydrophobic interactions, gravitational attraction, magnetic, capillary, and entropic forces. The consequence of these specific local interactions among the components, without external direction, is generally the formation of ordered state forms as the system approaches equilibrium, reducing its free energy.

While the range of symmetries or structures is somewhat limited, the promise of high throughput and low-cost processing over large areas implies that, at least in principle, processing methods that leverage self-assembly will have a central role in the development of advanced nanotechnology. For the most part, their application to OFs provided so far randomly arranged or poorly ordered nanostructures. However, merging highly ordered nanostructures and bottom-up nanofabrication on the OFs would have many advantages, from reproducibility of fabrication to enhanced light-matter effects.

Recently, we reviewed those nanofabrication methods, based on self-assembly, that have been used to create lab-on-fiber nanoprobes, covering papers published until the beginning of 2018 [4]. That collection of articles clearly indicated that decorating the OF with ordered patterns by exploiting the natural organization of matter, exactly in the same manner that nature does, is a challenging but achievable goal. Withstanding this, one of the main shortcomings in the use of self-assembly as a valuable cost-effective nanofabrication tool for lab-on-fiber platforms relies on limited versatility in terms of possible attainable geometries and from the natural occurrence of unwanted defects breaking the ordered pattern.

In this review, we focus on the importance of the "order" in patterns for photonic applications and specifically for lab-on-fiber platforms. To this aim, in Section 2, we highlight the basic phenomenological aspects associated with periodic and aperiodic ordered systems in photonics. The attempts made so far to decorate the OF with highly ordered nanostructures by bottom-up methods are briefly summarized in Section 3, with emphasis on very recent papers. Special attention is devoted to reporting on techniques enabling the enlargement of the set of attainable geometries and the removal of unwanted defects. The review ends with a brief summary of available tools to quantify the degree of order.

\section{The Importance of Order}

\subsection{Periodic Ordered Structures}

In photonics, periodically ordered structures are typically named photonic crystals (PCs) [5]. These periodic structures, whose length scale is proportional to the wavelength of light in the band gap, are the electromagnetic analogue of a crystalline atomic lattice. If the dielectric constants of the materials in the crystal are different enough, and the absorption of light by the material is minimal, then scattering at the interfaces can produce many of the same phenomena for photons as the atomic potential does for electrons. In particular, PCs 
may possess photonic band gaps, which are ranges of frequencies in which light cannot propagate through the structure.

In the last years, they have inspired a lot of interest in the scientific community, because they offer unprecedented opportunities for the realization of miniaturized optical devices [6-9]. The introduction of proper defects in the periodic medium in terms of geometrical or physical parameters modifications, acting as defect states inside the photonic bandgap, gives the resulting device interesting functionalities (Figure 1a). The defect formation breaks the symmetry pertaining to the "ordered pattern" and enables electromagnetic field localization and light flow manipulation [5]. The key feature of such defected structures relies on their unique guidance capability $[10,11]$ but also on the tuning or sensing opportunity which can be provided to the whole photonic device by acting on the physical and geometrical parameters at the defect location. The properties of PCs out of the plane of periodicity are investigated too. In particular, guided resonances, which are PC characteristic modes that couple to out-of-plane impinging external radiation, provide interesting sharp spectral features in dielectric PC slabs with potential applications in imaging and sensing [12].

The integration of metals with dielectric patterns allows the withstanding of surface plasmon resonances (SPR) and localized SPR. The characteristic enhancement at the metal surfaces can be judiciously exploited for ultrasensitive biological probes.

The translation of such photonic ordered structures on the OF platforms is part of the lab-on-fiber technology development [2,3]. In this regard, bottom-up methodologies based on self-assembly procedures provide a high production throughput without using high-cost microfabrication facilities. Unfortunately, these processes are still difficult to implement on unconventional substrates such as the OFs and the formation of ad-hoc defects is inherently limited. Remarkable examples of photonic structures self-assembled in a highly ordered fashion on the OF are reported in Section 3.

\subsection{Aperiodic Ordered Structures}

The concept of order is often erroneously restricted to the concept of "periodic" order [13]. In the last decades, the "order without periodicity" has emerged to properly describe an increasing number of complex systems. Namely, "quasicrystals" are materials or structures exhibiting long-range order and yet do not have translational periodicity. Quasicrystals permit symmetry operations forbidden in classical crystallography, for example, five-, eight-, ten- and 12-fold rotations.

Daniel Shechtman, awarded with Nobel Prize in 2011 for the discovery of quasicrystals, observed for the first time the quasicrystals in a diffraction experiment by electron microscopy in April 1982 [14] during his study of the structural morphology (icosahedral) of a rapidly solidified (Al, Mn) alloy [15]. The discovery spurred a lot of debate, but also intense activity aimed at synthesizing new quasicrystals. Within a few years, the icosahedral quasicrystals were joined by axial quasicrystals with decagonal and pentagonal symmetry [16,17], octagonal symmetry [18], dodecagonal symmetry [19] and enneagonal symmetry [20].

Later, quasicrystals were demonstrated in the soft matter too. Supramolecular structures of organic dendrimers [21] and tri-block copolymers [22] were synthetized. Colloidal inorganic nanoparticles were self-assembled into binary aperiodic superlattices with dodecagonal quasicrystalline order [23]. It is worth noting that the synthesis of quasiperiodic crystals in principle can be scaled up to micrometer feature sizes by a bottom-up approach too. Indeed, in 2008, micron-sized colloidal spheres were arranged into quasicrystalline arrays by using intense laser beams that create quasi-periodic optical standing-wave patterns [24].

Alongside the fabrication and discovery of different quasicrystals, the studies of phenomena exhibiting aperiodic arrangements of matter in space and/or time were spurred, and the basic knowledge gained from these studies has naturally extended to several research domains. 
The photonic properties of quasicrystals have been a subject of intense research. Many studies were devoted to assessing the phenomena that govern wave transport and interference in aperiodic systems [25]. It has been shown that photonic quasicrystals could exhibit photonic band [26,27] as well as light wave localization [28]. The frequencies where the bandgaps occur are not necessarily explained using single scattering and should therefore involve multiple scattering [29]. Aperiodic structures in two spatial dimensions combine long-range structural order with higher-order rotational axes (i.e., forbidden symmetries) leading to more isotropic band gaps, omnidirectional reflection spectra, and unusual localization of optical modes.

The possibilities of quasicrystals to support guided resonances have been the subject of a series of theoretical and experimental works. In a pioneering work from Ricciardi et al. [30], the transmittance response and the modal analysis of a photonic quasicrystal slab demonstrated the possibility to excite guided-resonance modes, and highlighted similarities and differences with the periodic case. In particular, it is shown that, as for the periodic case, guided resonances arise from the coupling of the incident plane-wave with degenerate modes of the photonic quasicrystal slab that exhibit a matching symmetry in the spatial distribution, and can still be parameterized via a Fano-like model. Further studies demonstrated the possibility to excite otherwise uncoupled guided resonances via the judicious introduction of symmetry-breaking point-defects [31] as well as the potential for both sensing and telecommunication applications [32].

Following such theoretical and numerical studies, in 2010, Ricciardi et al. provided the first experimental evidence of Fano-type guided resonances in aperiodically-ordered photonic quasicrystal slabs (Ammann-Beenker octagonal tiling) as shown in Figure 1c [33,34].

New designs based on the aperiodic ordered photonic structures were proposed for promising technological applications as well. Notomi et al. have studied the emission in aperiodically-ordered structures, considering a Penrosetype quasicrystal laser [35]. Mahler et al. demonstrated a quasi-periodic distributed feedback laser based on Fibonacci mirrors enabling directional output independent of the emission frequency and multicolor operation [36].

Nanoscale aperiodic surfaces were proposed for label-free biological sensing applications. The photon trapping arising from higher-order multiple scattering processes leads to a dense spectrum of highly complex structural resonances, i.e., colorimetric fingerprints, like those shown in Figure 1d, which can be captured with conventional dark-field microscopy and exploited to detect surrounding refractive index variations by means of imaging analysis techniques [37]. In particular, both the peak wavelength shifts of the scattered radiation as well as the environment-dependent spatial structure of the colorimetric fingerprints of aperiodic surfaces have already been utilized to detect the presence of nanoscale protein layers [38].

Quasicrystals were investigated for solar energy harvesting too. Light-trapping optimization by nanopatterning is in fact a crucial point to enhance the amount of light that can be absorbed by the device, especially in thin-film silicon solar cells, where the light absorbance can be a bottleneck of the photovoltaic process [39]. For example, an established approach to increase light trapping in thin-film silicon solar cells is the growth of transparent zinc oxide electrodes with a random pyramidal texture by means of chemical vapor deposition. This texture has already demonstrated exceptional light-trapping capabilities and is essential in overtaking the $10 \%$ conversion efficiency limit for single junction amorphous silicon solar cells [40]. Battaglia et al. made a comparison between the light-trapping efficacy of random nanopyramids and of periodic nanocavities with similar dimensional features (Figure 1e). By performing theoretical calculations on the basis of the two real geometries they predicted that periodic structures should outperform random textures, as they avoid scattering into lossy radiation channels. The external quantum efficiency of the two devices, however, was exactly the same, leading to the conclusion that the performance of thin-film silicon solar cells is currently limited by the trade-off between optical and electrical performance [41]. 

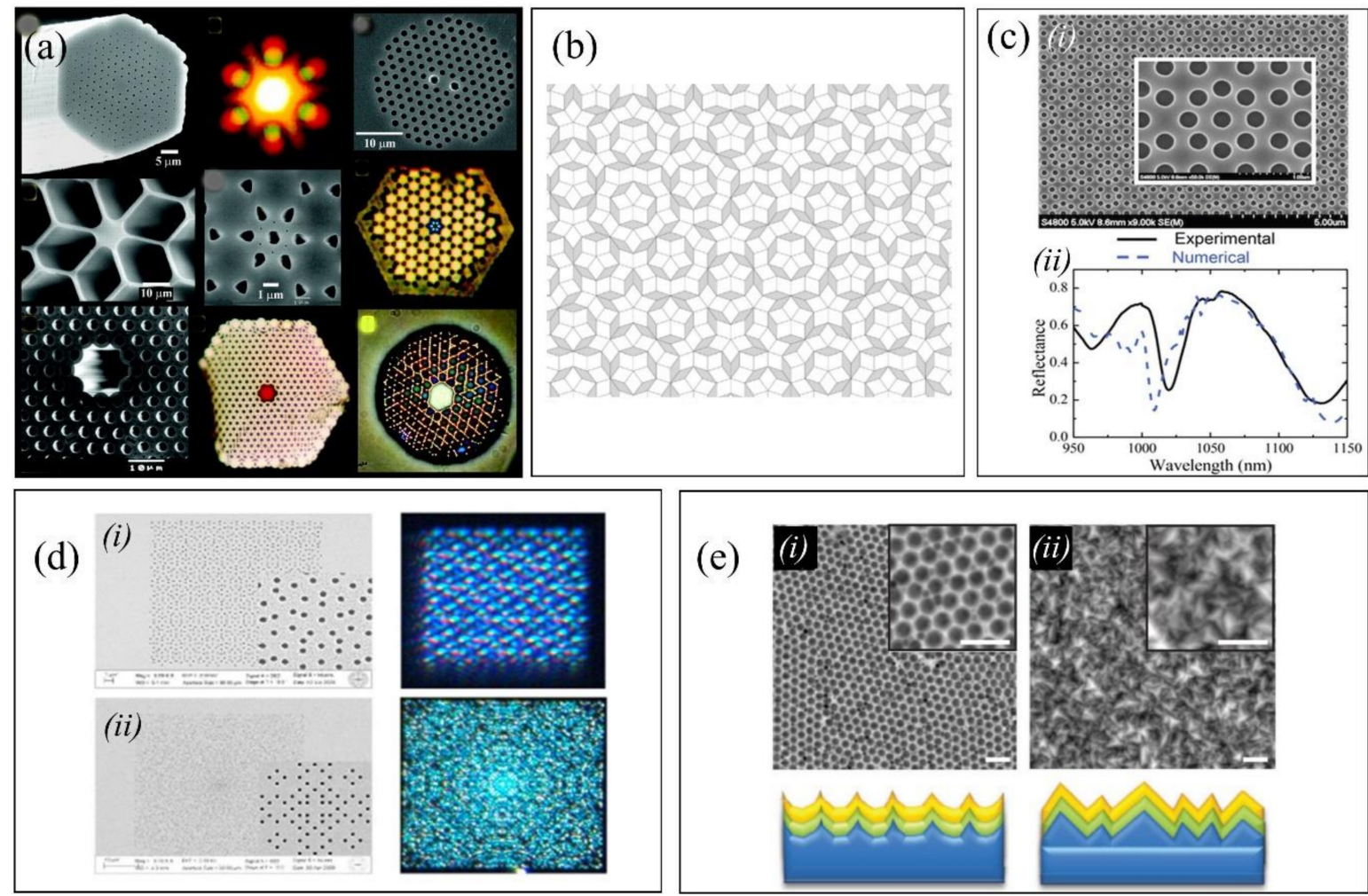

Figure 1. (a) Assortment of optical and SEM micrographs of photonic crystal fiber structures including proper defects in the periodic structure. Reproduced from Ref. [11] with permission from the 2003 American Association for the Advancement of Science. (b) The pentagonal Penrose tiling, which paved the way for the discovery of quasicrystals, is a self-similar pattern with 5-fold symmetry, long-range order and no translational periodicity. Reproduced from [14]. (c) SEM micrograph of an aperiodically-ordered photonic quasicrystal slab operating at near-infrared wavelengths (i) and measured reflectance response (ii), from which a Fano-type resonant lineshape is clearly visible, in good agreement with numerical simulations. Reproduced from Ref. [30]. (d) SEM images and colorimetric fingerprints of 2D aperiodic gratings: (i) Penrose lattice ( $(\mathrm{d}=400 \mathrm{~nm})$, and (ii) Gaussian prime lattice $(\mathrm{d}=300 \mathrm{~nm}$ ). Reproduced from Ref. [37]. (e) AFM images of periodic nanocavity array (i) and random pyramidal texture (ii), used to evaluate the light-trapping efficiency of protonic nanostructures in hydrogenated amorphous silicon thin-film solar cells (scale bars $1 \mu \mathrm{m}$ ), with the corresponding drawing of the cell on the bottom. Reproduced from Ref. [41] with permission from the 2012 American Chemical Society.

In a more recent study, Micco et al. showed that aperiodic tiling of photonic quasicrystals could potentially offer specific advantages to enhance light trapping capability through aperiodic patterned back reflectors in thin film solar cells by fully exploiting the additional degree of freedom and the richness of states offered by quasicrystals [42].

\section{Bottom-Up Fabrication of Periodic Structures on OFs}

This section provides a concise description of the main bottom-up fabrication approaches to produce highly ordered nanostructures and their application attempts on the OF. For the most part, bottom-up nanofabrication relies on self-assembly processes. Nature-inspired processes have shown to be an extremely useful and versatile tool for building nanostructures, hierarchically ordered structures and sophisticated micro- and nanopatterns, and therefore most of them have already been widely applied to OFs [4].

However, self-assembly often produces randomly arranged or poorly ordered structures, especially on large scale. The $\mathrm{OF}$ is an unconventional substrate for nanopatterning. According to the specific position where the patterned element is introduced (on the tip, inside a portion of the core, around the lateral surface) the pattern formation is limited by several aspects such as the curved topography, the high aspect ratio, the presence of tiny cavities, etc. Definitively, the creation of reproducible ordered patterns on OF is still 
a major challenge. To what extent such disorder (the number of unwanted defects as far as the deviations from the desired pattern) affects the overall device functionality is a key aspect settling the boundary between a significant technology shortcoming and specific characteristics of a technological approach.

In this section, we briefly report the methods used so far to create self-assembled ordered structures on the OF, whilst in the following, we will focus on methods to systematically assess the degree of order. At the end of this section, the main self-assembly methods applied to OFs are recalled and summarized in Table 1.

\subsection{Colloidal Self-Assembly}

For the greatest part, the processes employed so far to accomplish ordered patterns on OF are based on the self-assembly of colloidal particles. In fact, the auto-organization of colloids naturally brings to the formation of compact ordered structures. If the building blocks are monodispersed nanobeads and the process is controlled enough in order to minimize defect formation, the result is a crystal-like lattice, which represents the starting structure to obtain different nanopatterns.

Self-assembly can occur directly on the fiber, as well as on a different substrate and then transferred on the fiber. In the latter case, the preferred substrate is water surface, which is easy to clean, and allows for easy transfer to any substrate. The generally used method for the assembly at the air-water interface consists of preparing a hydroalcoholic colloidal dispersion and depositing it on the water surface through a piece of a silicon wafer or plasma-treated glass slide partially immersed in the water, but other set-ups are also possible. The resulting photonic structure can be 3D PCs (where multilayer assemblies are packed), inverse opals (where the 3D assembly is first infiltrated with a different material than the one constituting the spheres, and then the spheres are removed by dissolution), 2D photonic crystals (2DPCs), and its more popular variations gathered under the name of nanosphere lithography (NSL).

The first attempt to integrate colloidal crystals with the OF dates back to 2005 when Li et al. coated a single-mode OF with a monolayer of silica beads [43] by an isothermal heating evaporation method. A similar procedure was adopted by Lin et al. [44]. They used a microstructured OF featuring two $110 \mu \mathrm{m}$ large holes. The fiber was immersed in a vial containing a poly(methylmethacrylate) (PMMA) colloidal suspension in water. The size of the colloidal PMMA spheres was $300 \mathrm{~nm}$. A face-centered-cubic lattice-type structure was achieved inside the twin-hole $O F$ for a length of $2.5 \mathrm{~cm}$.

Yan et al. fabricated the colloidal crystal directly on the tip of an OF [45]. Preliminarily, wet chemical etching technique was employed to fabricate a cavity in one side of the fiber while keeping the coating intact. The fiber was immersed with the cavity upward in the PMMA colloidal dispersion (microspheres of $690 \mathrm{~nm}$ diameter in water/ethanol mixture). After $2-3 \mathrm{~h}$ at $50^{\circ} \mathrm{C}$, the colloidal PC self-assembled on the tip by isothermal heating evaporation [45]. Successively, a colloidal crystal of polystyrene (PS) nanospheres with a $200 \mathrm{~nm}$ mean diameter was self-assembled onto the tip of a UV-VIS multimode OF by means of a dip-coating approach. The obtained fiber probes were used for label-free biosensor applications and their performances were evaluated using the highly stable streptavidin-biotin binding [46].

Self-organized colloids provide also a large-scale template for infiltrating different materials and obtaining inverse opals. In 2005, Moon et al. exploited a cylindrical shell of colloidal crystals assembled around an OF by a dip-coating method in order to create microporous arrays of interconnected air cavities [47]. A UV-curable polymer precursor was infiltrated and polymerized into the interstices of the colloidal crystal template and the microporous polymer structures around the OF were obtained by dissolving the PS spheres into toluene. Similarly, the process was demonstrated by using a silica colloid with $2 \mu \mathrm{m}$ diameter. Here, in order to obtain the inverse structure, after the infiltration and cure of the polymer precursor, the silica spheres and the fiber itself were removed simultaneously 
by chemical etching in a diluted hydrofluoric acid solution, leaving an inverted colloidal crystal with hollow cores.

The formation of inverse opals on the internal and external surface of a glass capillary was similarily carried out by $\mathrm{Ni}$ et al. [48], employing a sol-gel co-assembly method previously demonstrated on planar substrates[49].

The same authors performed also a systematic study aimed at the integration of opal and inverse opal films onto bundles of OFs [50]. By using isothermal heating evaporation induced self-assembly and sol-gel co-assembly methods, respectively, they realized opal films and silica inverse opal films on several optical fibers simultaneously. The transaltion of the technique on multiple fibers (i.e., bundle) outlined the potential impact of such self-assembly techniques for the mass production of OF devices. As proof of concept, the fabricate probes were tested as humidity sensors, exploiting the effective refractive index changes due to the humidity concentration variations [51].

Recently, Pisco et al. used the self-assembly of a monolayer colloidal crystal as a basic building block to obtain different periodic structures onto the OF tip [52]. Specifically, PS nanospheres (with diameters ranging from 200 to $1000 \mathrm{~nm}$ ) were self-assembled at the air-water interface into ordered hexagonal close-packed arrays and then used as basic building blocks to obtain different periodic structures after the transfer onto the OF tip (Figure 2a). The basic close-packed array (CPA) monolayer covered by a gold conformal layer was demonstrated to work as efficient surface-enhanced Raman spectroscopy (SERS) probe $[53,54]$. Similar structures were obtained by adding a plasma etching step before the metal deposition in order to reduce the nanosphere diameter maintaining the initial pattern period (SA, sparse array) [52]. The nanospheres monolayer, arranged either in CPA or SA fashion, can be used as a mask to pattern the underlying substrate too. After metal deposition, indeed, the nanospheres, removed from the surface by sonication, leave behind metal triangles in the regions corresponding to the void volumes between nanospheres (CPA-SR) or holes in a metal substrate corresponding to the etched nanospheres (SA-SR). The plasmonic properties of such structures were efficiently exploited to create OF probes for label-free bio-sensing and SERS applications.

By adopting a similar fabrication approach, Chang et al. realized nanotriangles patterns (CPA-SR) on fiber tip using nanospheres with $450 \mathrm{~nm}$ diameter and different film thicknesses as SERS substrates. The probe functionality was verified by measuring the Raman spectrum of a mixed solution of diethylthiatricarbocyanine and crystal violet [55]. Antohe et al. exploited the same sensing configuration, featuring a period of $500 \mathrm{~nm}$, as a plasmonic probe by investigating the effect of different gold thicknesses on the surrounding refractive index sensitivity [56]. Xia et al. reported on the SERS performances of a CPA-SR probe using Rhodamine 6G (R6G) as target analyte (Figure 2b). In order to improve the SERS enhancement factor, they proposed a hybrid graphene/Au nanotriangle structure capable of exploiting the SERS chemical effect associated with the charge transfer between graphene and the target molecules [57]. In addition, they systematically investigated the optical coupling between the fiber and the Raman spectrometer (schematized in Figure 2(b-i)) by optimizing the intensity of Raman signal passing through the fiber probe. A similar optimization was carried out in refs $[53,54]$ for CPA sensing probes.

Very recently, Liang et al. demonstrated a plasmonic nanohole-patterned (SA-SR) multimode OF probe for real-time monitoring the specific binding of proteins [58]. In the same year, Liu et al. proposed an alternative method to achieve similar structures by combining colloidal sphere self-assembly with ultraviolet (UV) nanoimprint lithography [59]. The whole fabrication process is shown in Figure 2c. The PS CPA was firstly formed on a glass substrate. Then, a thin layer of UV-curable film was coated on the OF tip. The PS CPA was embedded in the UV-curable resin by pressing it onto the OF end face onto the OF end face. Finally, after dissolving the PS spheres and depositing a thin metal film, a nanocavity-patterned fiber probe was achieved. From the morphological images, the degree of order was limited to a short range. Nonetheless, the fabricated fiber optic samples were successfully employed as a SERS probe and label-free biosensor. The same 
authors exploited this technique to create a Pd pattern of CPA-SR around a capillary tube for hydrogen sensing [60].

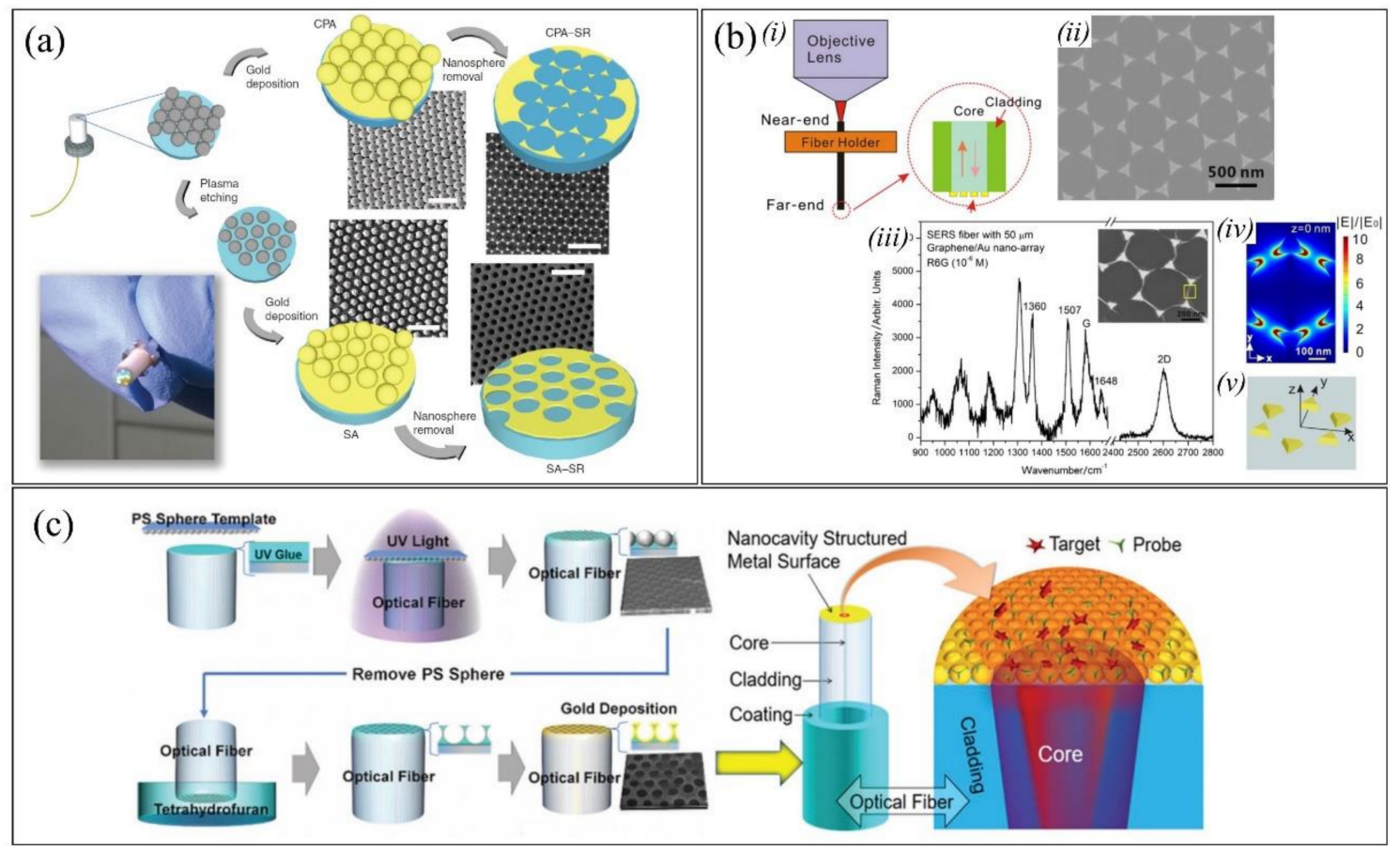

Figure 2. (a) Fabrication procedure for creating four different fiber tip nanoprobes and SEM images (scale bar $=3 \mu \mathrm{m}$ ) of real samples obtained using 1- $\mu \mathrm{m}$ nanospheres, and photograph of a fiber tip showing an iridescent nanopattern. Reproduced from Ref. [52]. (b) Fiber probe with hybrid graphene/Au nano-triangle nanostructuration: schematic diagram of the experiment setup (i), SEM image of Au nano-triangle array (ii), R6G Raman spectrum obtained by fiber probe (iii), electric field magnitude mapping along $x-y$ plan for $z=0 \mathrm{~nm}$ (iv) and schematic model of Au triangle array (v). Reproduced from Ref. [57] with permission from 2017 WILEY-VCH Verlag GmbH and Co. KGaA, Weinheim, Germany. (c) Fabrication procedure for the nanocavities-patterned fiber probe obtained by combining colloidal assembly and UV nanoimprinting, which includes the coating, patterning, and metallization of the cavities on the fiber end face. Reproduced from Ref. [59] with permission from 2019 WILEY-VCH Verlag GmbH and Co. KGaA, Weinheim, Germany.

\subsection{Other Self-Assembly Methods}

Besides colloidal self-assembly, a few other approaches guided by an auto-organization phenomenon are capable of generating highly ordered patterns: breath figure formation and directed self-assembly.

\subsubsection{Breath Figures}

The formation of breath figures is a natural phenomenon observable anytime water vapors condensate on a cold surface. The fog formed on a glass window while breathing on it, when viewed under a microscope appears as an assembly of closely packed micrometric water droplets [61]. The most typical application of this simple phenomenon in nanotechnology consists in generating the fog on a polymeric solution by blowing some humid gas toward it, so that at the end of the process the water droplets leave their imprint in the polymer film, generating a microporous film with the characteristic honeycomb structure, known as breath figure array [62]. By controlling the main parameters of the process (type of polymer and solvent employed, gas flow rate, humidity content) highly ordered patterns can be easily obtained, even on a large scale [63].

By analogy, breath figures can be assimilated to the assembly of 2DPCs at the air/water interface, where the interface considered here is that between polymer solution and air, and the packing objects are not solid particles but water droplets. In breath figures, in fact, 
water droplets are stabilized by the polymer molecules preventing their coalescence, and therefore they can move and pack themselves as long as the polymer solution is still in liquid form. When the solvent evaporates leaving a solid polymer film, water droplets are frozen in their positions; as soon as also water evaporates, its imprint remains on the film. Typically, a volatile and non-water-mixable solvent such as chloroform or carbon disulfide is employed to dissolve the polymer. The water droplets naturally form on the cast polymer solution by water condensation, due to a local temperature drop triggered by the solvent evaporation. It is also possible to manage this phenomenon more accurately by regulating the substrate temperature using a Peltier controller.

The potentiality of this technology is extensive, especially in terms of production speed: fabricating a colloidal 2DPC takes tenths of minutes to hours; making a pattern by breath figures takes a few seconds to $2-3 \mathrm{~min}$. Thanks to its extreme simplicity and fast realization, the breath figure process has been applied in materials science in a great number of studies and for different applications [64-71]. For nanofabrication purposes, breath figure arrays can also represent the starting structure from which different kinds of patterns can be developed by further fabrication steps such as electrodeposition [72], pyrolysis [73], or dry etching [74].

Pisco et al. recently used the breath figures to decorate the OF tip with photonicplasmonic patterns, and their work remains the only example so far [75]. The nanofabrication approach is depicted in Figure 3. This work demonstrates that breath figure formation can be adapted to work on the OF. The metallo-dielectric pattern obtained by thermal evaporation of a thin gold layer on the top of the honeycomb film allowed the authors to develop an OF probe capable of detecting refracting index unit (RIU) variations with a sensitivity of up to $2300 \mathrm{~nm} / \mathrm{RIU}$, surpassing the performances of most analogue sensing probes fabricated by using more technically demanding techniques. The possibility of modulating the lattice period by controlling the casting conditions is also demonstrated.
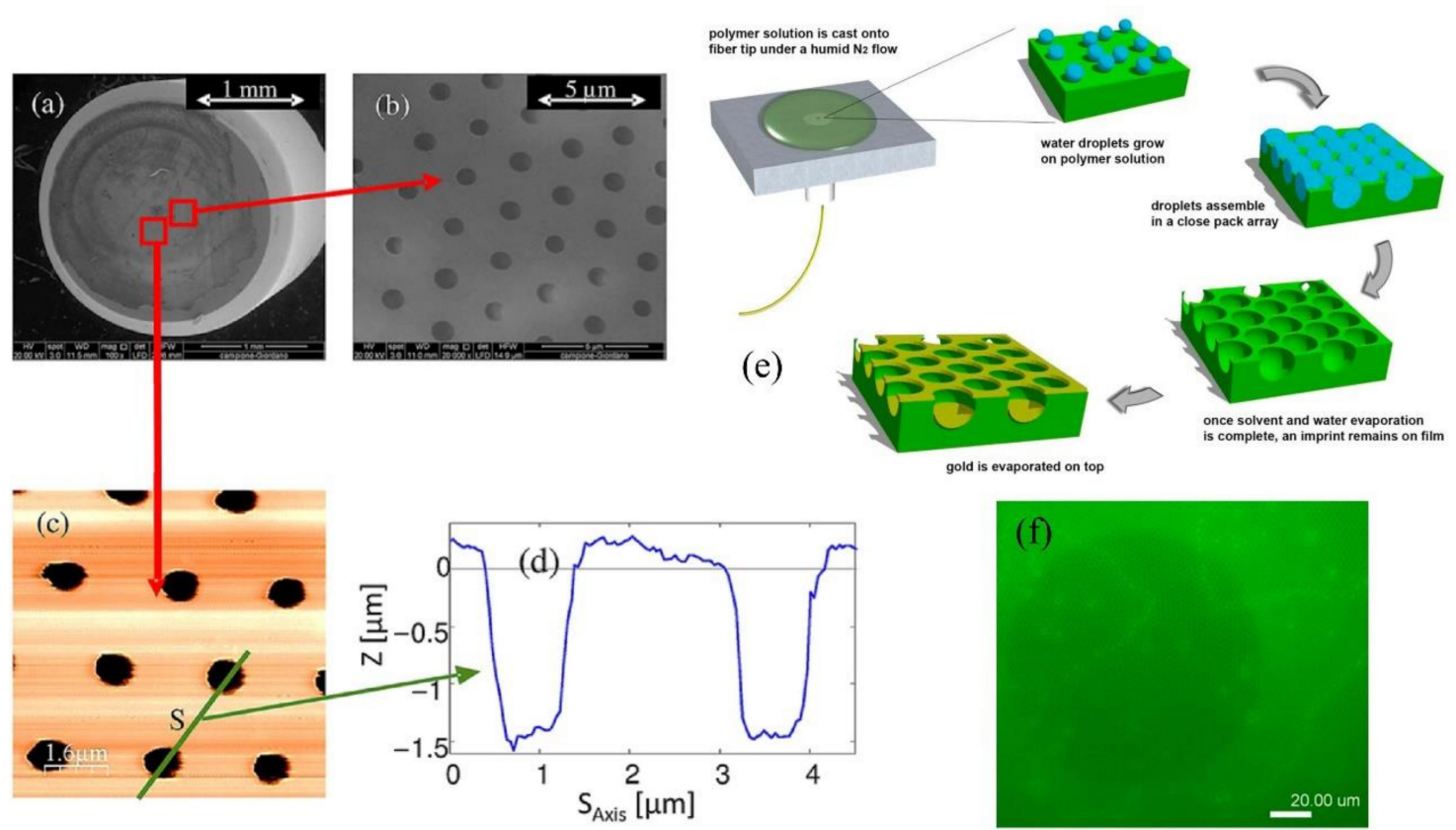

Figure 3. Breath figure approach to periodic pattern nanofabrication on the optical fiber. (a) SEM top view of the optical fiber decorated with the photonic-plasmonic pattern obtained by breath figure approach; (b) magnified SEM image; (c) AFM topography; (d) z profile along S line in (c); (e) schematic overview of the fabrication process on the optical fiber; (f) fluorescence microscopy image of the honeycomb film realized on the optical fiber, showing the highly ordered pattern on an extensive area (the darker round area delimitates the fiber facet). Reproduced from Ref. [75] with permission from the 2014 American Chemical Society. 


\subsubsection{Directed Self-Assembly}

Nanofabrication methods based on directed self-assembly (DSA) utilize a template to guide the self-assembly process with the aim of minimizing the defect formation and promoting a long-range order organization. This technology has reached a considerable advancement level in the semiconductor and data-storage field, where DSA is one of the leading candidates for next-generation lithography. In this case, in fact, the spontaneous orientation of block-copolymers (BCPs) occurring by phase separation (giving rise to lamellae or cylinder arrays) is directed by a pre-patterned substrate [76,77]. As a result, $\mathrm{BCP}$ long-range organization is promoted (Figure 4a) and the pitch of the final pattern can be reduced towards the sub-10 $\mathrm{nm}$ limit $[78,79]$. Since the pre-patterning is often fabricated by conventional lithography methods, we can consider DSA as a combination of top-down and bottom-up approaches. Similar to NSL and BF approaches, the polymeric pattern can be transformed by further fabrication steps like selective etching or metal deposition into metallo-dielectric periodic patterns suitable for a variety of photonic applications.

Table 1. Comparative summary of the different self-assembly techniques applied to optical fibers.

\begin{tabular}{|c|c|c|c|c|}
\hline Main Methods & Structures & $\begin{array}{c}\text { Feature Sizes } \\
\text { (for "Ordered" Patterns) }\end{array}$ & Symmetry & $\begin{array}{c}\text { Applications } \\
\text { (Experimentally Validated) }\end{array}$ \\
\hline $\begin{array}{l}\text { Colloidal Self-assembly } \\
\text { (SA)—basic } \\
\text { method [43-48,50,51] }\end{array}$ & $\begin{array}{l}\text { 2D and 3D, opal and inverse } \\
\text { opal, photonic crystals }\end{array}$ & $\begin{array}{l}\text { from hundreds of nm up to a } \\
\qquad \text { few } \mu \mathrm{m}\end{array}$ & hexagonal & $\begin{array}{l}\text { Refractive index and RH } \\
\text { sensors [51], label-free } \\
\text { biosensors [46] }\end{array}$ \\
\hline $\begin{array}{l}\text { Nanosphere lithography } \\
\text { (NSL) [52-60] }\end{array}$ & $\begin{array}{c}\text { Closed packed array of } \\
\text { nanospheres, sparse array of } \\
\text { nanospheres, triangular } \\
\text { nanoislands, honeycomb } \\
\text { holey metallic film }\end{array}$ & $\begin{array}{l}\text { from hundreds of nm up to a } \\
\qquad \text { few } \mu \mathrm{m}\end{array}$ & hexagonal & $\begin{array}{l}\text { Refractive index [56], biological } \\
\text { sensors [58], SERS probes } \\
\text { [52-55,57], hydrogen sensor [60] }\end{array}$ \\
\hline Breath figures (BF) [75] & $\begin{array}{c}\text { Microporous } \\
\text { metallic-dielectric films }\end{array}$ & $\mu \mathrm{m}$ scale & hexagonal & Refractive index [75] \\
\hline $\begin{array}{l}\text { Direct self-assembly } \\
\text { (DSA)—hybrid } \\
\text { method [80-83] }\end{array}$ & $\begin{array}{l}\text { Concentric rings, spiral and } \\
\text { chiral nanostructures, cluster } \\
\text { array of nanoparticles }\end{array}$ & from nm scale up to $\mu \mathrm{m}$ scale & linear, circular, hexagonal & SERS probe [83] \\
\hline
\end{tabular}

DSA of BCPs can be also adapted to produce patterning geometries that would be impossible to obtain by natural BCP self-organization. For example, Choi et al. employed a circular template as a guiding pattern for a poly(styrene-block-dimethylsiloxane) (PSb-PDMS) [80]. Depending on the presence of a notch in the circular template, either concentric rings or spiral nanostructures can be obtained from the cylindrical organization of the BCP (Figure $4 \mathrm{~b}$ ). Moreover, the authors demonstrated that they could control the $2 \mathrm{D}$ chirality of the spirals by adjusting the position of the notch. Considering that $2 \mathrm{D}$ and 3D chiral nanostructures have many potential applications in the sensing of molecular chirality or as chiral metamaterials, adapting this DSA process to the OF tip would be particularly advantageous.

Besides BCPs, DSA can also be applied to nanoparticles [81]. As for BCPs, nanoparticle organization can be guided by a substrate bearing a pre-formed pattern, so that uniform and defect-free monolayers of colloidal particles can be obtained. In the example of Figure $4 \mathrm{c}$, the authors study the assembly of PS nanoparticles in confined geometries fabricated by direct laser writing lithography. By simple spin-coating of a colloidal solution over substrates patterned with linear, hexagonal and circular structures, single-grain crystals with uniform orientation and regular shape over a large area were achieved [82]. Another option is to employ the periodic nanopattern of BCPs as a template, to guide the nanoparticle assembly. This approach was followed by Yap et al. to decorate the OF tip with cluster arrays of Au nanoparticles [83]. Poly(styrene-block-2-vinylpyridine) (PS-b-P2VP) was spin-coated on the chosen substrate (either a piece of silicon wafer or the OF tip) to obtain an array of reverse micelles (Figure 4(d-ii)). Then, the external surface of this film was plasma etched, to expose the positively charged part of the micelles to the outside. By immersion in a colloidal solution of Au nanoparticles, the micelles were selectively coated by the electrostatic assembly. The resulting OFs decorated by periodic 
patterns of clusters composed of randomly aggregated metal nanoparticles were used as SERS probes (Figure 4(d-iv)). This investigation demonstrated that ordered patterns with plasmonic properties can be obtained on flat substrates, while the process applied to the OFs still needs some optimization to enhance the long-range order of the nanostructures.

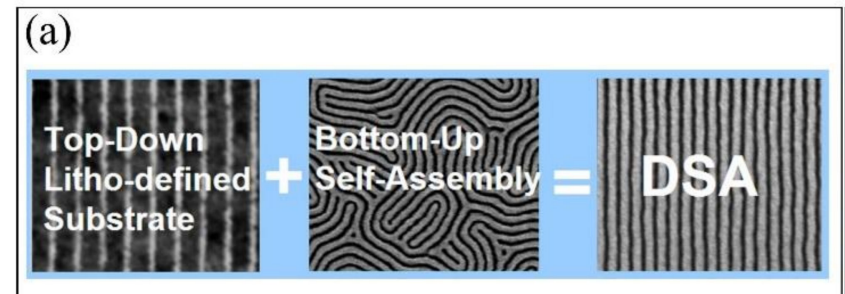

(b)

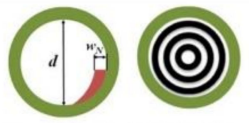

(i)

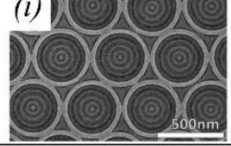

(c) (i)

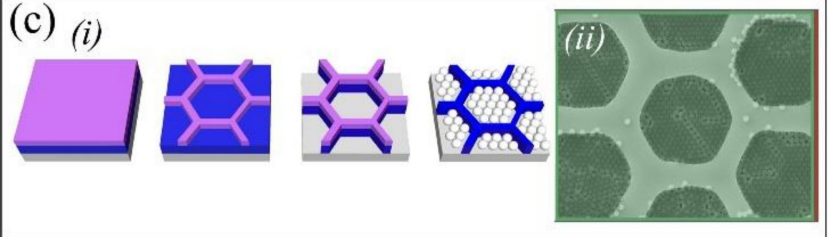

(d)
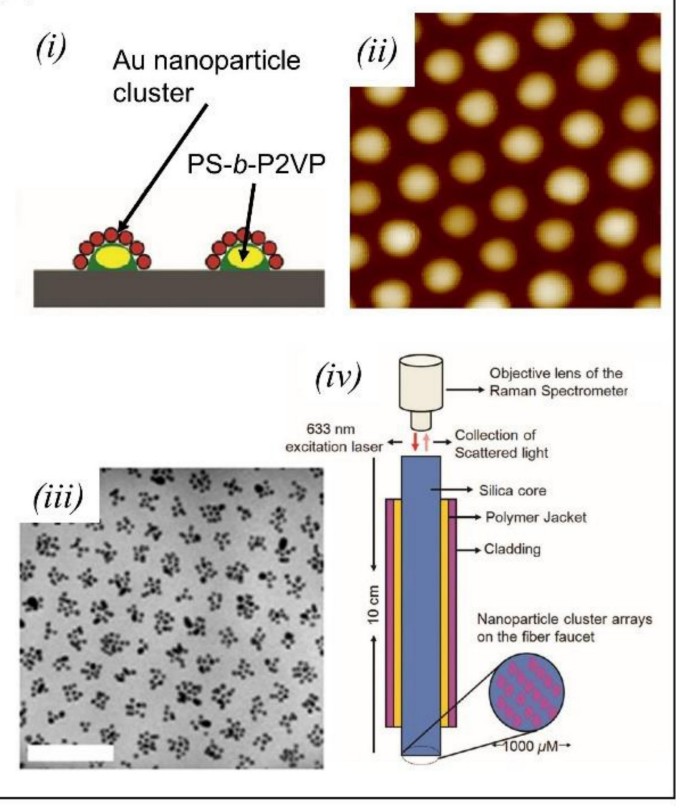

Figure 4. (a) Representation of the directed self-assembly (DSA) approach: the combination of block-copolymer selfassembled into organized nanoscale structures with lithographically defined structures acting as guiding patterns, produces highly regular patterns of lines/spaces. Reproduced from Ref. [79] (b) Schematic (top) and experimental (bottom) examples of concentric rings (i) and spirals of right-handed (ii) or left-handed (iii) chirality, formed by a block copolymer selfassembled in circular (i) and notched (ii,iii) templates. The template diameters in (i-iii) are $350 \mathrm{~nm}$. Reproduced from Ref. [80] with permission from 2017 IOP Publishing Ltd. (c) Schematic representation of the lithographic process to obtain a hexagonal pattern for DSA of colloidal particles (i) and SEM micrograph of the assembled particles in the hexagonal pattern. Reproduced from Ref. [82]. (d) Schematic representation of gold nanoparticle clusters grown around PS-b-P2VP inverse micelles assembled on a substrate (i); AFM image of the array of PS-b-P2VP micelles (ii); SEM micrograph of the array of Au nanoclusters resulting from the decoration of the BCP micelles (iii); schematic representation of the SERS analysis setup including the patterned optical fiber probe (iv). Reproduced from Ref. [83] with permission from the 2012 American Chemical Society.

\subsection{Post-Processing Methods to Increase the Order}

Nanofabrication methods based on self-assembly processes are naturally inclined to include a certain number of defects in the assembled structure. Sometimes the presence of defects may be a benefit for the function of the nanostructure. For example, the controlled introduction of defects into the PC structure allows for additional tailoring of light propagation (Figure 5a) [84]. Most of the time, however, the material scientist is called to minimize the number of defects produced by the chosen manufacturing process. This can be achieved by accurate control of the key parameters of the process, so that the self-assembly phenomenon is piloted to produce always the same periodic structure. These may include environmental parameters ( $\mathrm{T}$, relative humidity $(\mathrm{RH})$, light exposition) purity of materials used, homogeneity of size and shape in case of nanoparticle assembly, process speed-rate, and extension of the patterning surface.

Besides using rigorous operational precautions, it is sometimes possible to increase the order degree of a self-assembled periodic pattern by applying a post-processing treatment. 
Thermal treatments, for example, can be effective in increasing the order when selfassembling elements organize themselves to reach their thermodynamic equilibrium. As illustrated in the previous paragraph, DSA is a clear example in this contest. BCP thin films, generally produced by spin or blade coating, are partially organized in short-range order domains. A post-processing treatment consisting in heating the $\mathrm{BCP}$ thin film to temperatures above the glass transition temperatures of all blocks induces the long-range order guided by the pre-pattern present on the substrate [85]. This happens because temperature increases the mobility of the crystallizing elements (the polymer blocks) facilitating reorganization into thermodynamically favored structures.

Besides temperature, solvent vapor annealing is frequently used to modulate the orientation and increase the order of BCP films. When the thin film is exposed to solvent vapors, the solvent adsorption promotes the formation of a swollen and mobile polymer layer at temperatures well below the highest glass transition temperature of the system. The choice of solvent and its optimal concentration in the ambient surrounding the film are key factors for the success of the annealing. For example, in a detailed study on the self-assembly of poly(styrene)-block-poly(lactide) thin films, Baruth et al. determined the optimal tetrahydrofuran (THF) concentration and vapor annealing time to obtain films with hexagonally close-packed perpendicularly aligned cylinders traversing the entire thickness of the film, low defect densities, and extended order [86].

Another reported method to increase the order of a periodic pattern is mechanical rubbing. This post-processing treatment is widely used in liquid crystal orientational epitaxy, for the alignment of polymeric layers which guide the orientation of liquid crystal molecules [87]. Besides liquid crystals, mechanical rubbing can also be applied to the alignment of different materials such as anisotropic nanocrystals [88], nanowires [89], and conjugated polymers [90], resulting in improved material properties like polarized photoluminescence and electric conductivity.

However, mechanical rubbing is not limited to the alignment of anisotropic particles or molecules within a direction. Interestingly, Jeong's group showed that it is possible to obtain highly ordered 2DPC by rubbing dry PS spheres [91]. The assembly process consists in rubbing the nanospheres between two pieces of PDMS elastomer. This randomly directed friction enables the particles to roll one on the other until they pack themselves in a monolayer (Figure $5 \mathrm{c}$ ). The process bases its success on the fact that the particle-substrate adhesion energy must be larger than the particle-particle attraction energy and the external shear force applied to the aggregate must be transferred to all particles in the aggregate. In other words, the particles must stick preferentially to the substrate than to themselves. At the above condition, once the particles stick with the substrate, it is difficult to be separated out again. In this way, it is feasible to break the multilayered agglomerates and form two monolayers of small and randomly oriented crystals, remaining on each elastomer surface. Then, in order to increase the size of the colloidal crystals, a second rubbing process, this time following a precise direction, must be applied repeatedly back and forth. The result of this post-processing treatment is the formation of large-scale and highly ordered 2DPCs. By testing different rubbing conditions, the authors could determine the optimal parameters in terms of rubbing speed and force (Figure 5(c-iii)). The possibility of assembling spheres of different materials (PS, silica, PMMA) as well as of using different kinds of rubbing elastomers are also demonstrated. Given the ability to increase the long-range order of the assembly and minimizing the crystal defects, this process is very appealing with respect to the classical assembly of colloidal dispersions used in NSL. The only limitation is that the dry process works on elastomeric surfaces only; however, coating any kind of substrate (glass, silicon) with a thin layer of PDMS is sufficient to perform the assembly. 


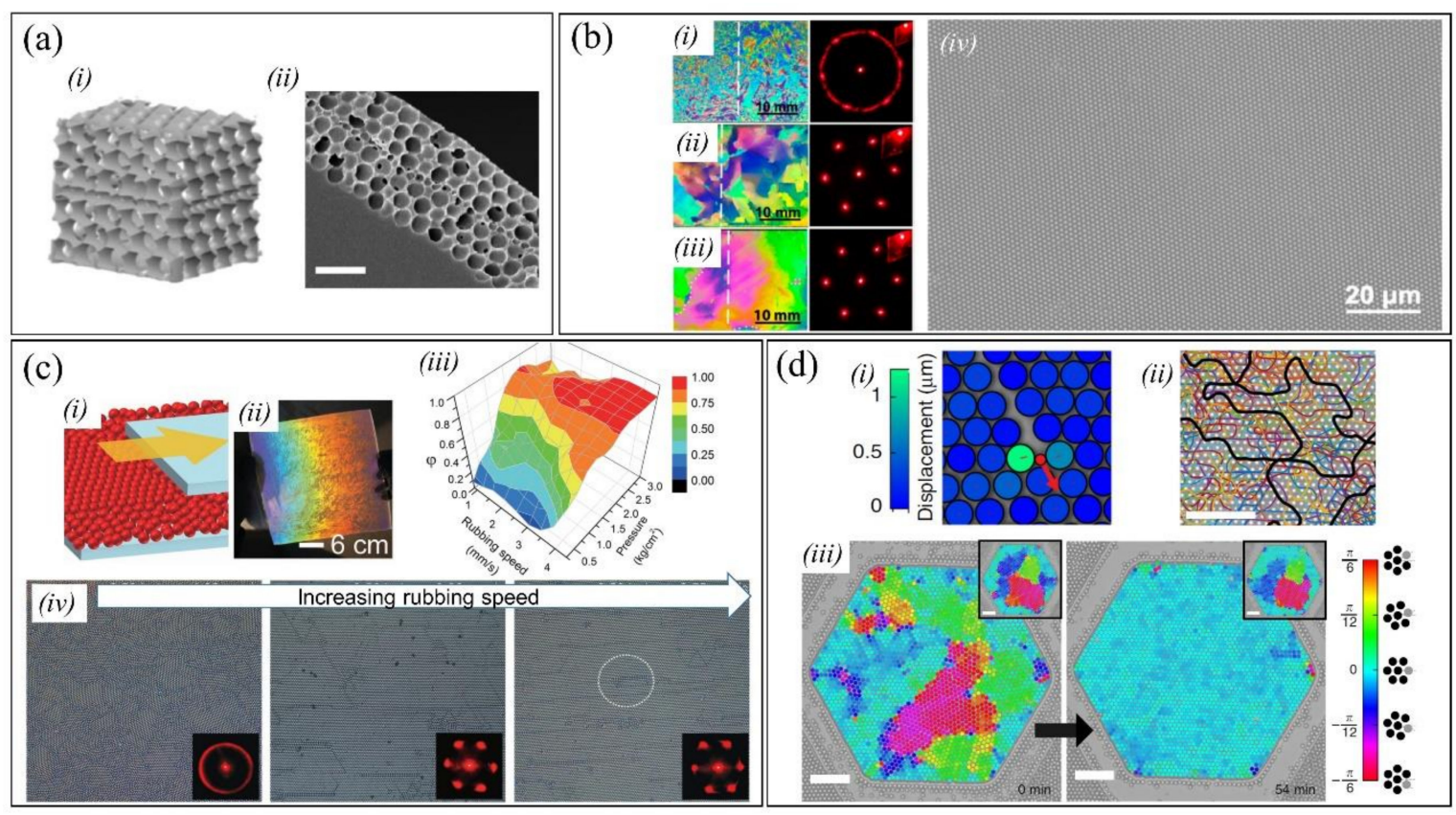

Figure 5. (a) Schematic representation (i) and cross-sectional SEM image (ii) of a silk fibroin inverse opal with an engineered photonic bandgap realized by introducing a planar defect layer of smaller cavities in the middle of six layers of bigger pores (scale bar, $1 \mu \mathrm{m}$ ). The introduction of this defect modifies the photonic band gap. Reproduced from Ref. [84] with permission from the 2018 Royal Society of Chemistry. (b) Optical image of polystyrene colloidal crystals before (i) and after (ii and iii) the gas-flow-assisted melting and reorganization treatment, showing the increase of crystal domain sizes up to several millimeters. The enhancement of periodic order is confirmed by the corresponding laser diffraction patterns. An example of defect-free colloidal crystal obtained by this process is also shown (iv). Reproduced from Ref. [92] with permission from the 2014 American Chemical Society. (c) Closely packed particle monolayers prepared by rubbing dry microspheres on elastomeric surfaces. Schematic showing the rubbing process (i) and the resulting monolayer assembly of polystyrene $(1 \mu \mathrm{m}$ in diameter) beads on the entire surface $(20 \mathrm{~cm} \times 20 \mathrm{~cm})$ of a PDMS substrate (ii); variation of degree of orientation $(\phi)$ of the colloidal crystal according to the rubbing speed and pressure determination of the optimal conditions of the pressure (iii); in-plane grain growth of the monolayer particle assembly produced by unidirectional rubbing at increasing rubbing speed from left to right, and corresponding laser diffraction patterns (inset) (iv). Reproduced from Ref. [91] with permission from 2019 WILEY-VCH Verlag GmbH and Co. KGaA, Weinheim, Germany. (d) Accelerated annealing process by self-propelled microswimmers. Calculated individual trajectories of self-propelled particles in an ordered monolayer (i); modeled displacement of colloids induced by a swimmer (red dot, the arrow indicates the direction of motion) (ii); optical microscopy of a monolayer of passive silica beads containing a small fraction of active intruders (not visible at this magnification) before (left) and after (right) the accelerated annealing process, showing the elimination of most of defects (the color-code indicates the local orientational field of the hexagonal structure) (scale bars, $50 \mu \mathrm{m}$ ) (iii). Reproduced from Ref. [93].

A different approach to increase the order of 2DPCs has been proposed by Meng and Qiu [92]. In this process, the monolayer colloidal crystal is formed by assembling the PS spheres at the air/water interface as described by many previously reported papers. The only difference is that a small amount of surfactant is introduced into the water phase to reduce the interaction energy density between water and PS particles, thereby the lateral attraction between PS particles. When the water surface is full of colloidal particles and the monolayer becomes "frozen" on the water surface, a compressed nitrogen flow is directed towards it, so that the "crystallites" are "melted" and then recrystallized. This "melting"-recrystallization process is carried on like the annealing treatment in molecular crystallization to eliminate defects (Figure $5 b$ ). A growth front of ordered 2DPC is generated and moved forward with the nitrogen flow by carefully moving the nitrogen nozzle along the surface, until 2D hexagonal colloidal single crystals of centimeter size are obtained. 
An innovative way to achieve a local reorganization of the colloidal crystal was recently proposed by Palacci and his co-workers [93]. The idea under this study is to exploit the motion of self-propelled particles to move some of the microspheres packed in a colloidal crystal from their position, so that the particles in the wrong position can rearrange, removing the defect (Figure 5d). As a result, the degree of order of the 2DPC highly increases. In this system, which represents the proof-of-concept of controlling a selfassembled structure by active particles, polycrystalline monolayers of $5 \mu \mathrm{m}$ silica spheres are assembled by sedimentation on the bottom of a microfluidic apparatus consisting of hexagonal cells filled with solvent (Figure 5(d-iii)). The propellers are light-activated microswimmers of $2 \mu \mathrm{m}$-diameter consisting of a hematite $\left(\mathrm{Fe}_{2} \mathrm{O}_{3}\right)$ portion protruding out of a polymer particle. Under UV light, the photocatalytic hematite triggers the local decomposition of the hydrogen peroxide contained in the solution, creating a gradient that sets the swimmer into motion (Figure 5(d-ii)). Since the speed of the microswimmers is tuned by light intensity, it is possible to operate on the system different reorganization cycles (as in thermal annealing) and even local reorganization by exposing it to light patterns, obtaining a spatial control of the annealing.

\section{Methods for Order Quantification}

The nanofabrication of ordered patterns by bottom-up approach besides needing a suitable assembly technique requires tools for evaluating and quantifying the order. As already mentioned, in fact, defect-free periodic structures are practically impossible to obtain by self-assembly, therefore the effectivity and reproducibility of the chosen processing method, as well as the comparison between different samples can only be achieved by defect analysis and order quantification. Moreover, the sensing performances will likely depend on the uniformity and degree of order of the nanostructure, especially when the objective of the nanofabrication is an OF probe. In this prospective, order quantification can correlate the structural quality with the optical quality and therefore con provides a prediction of the functionality of the sensor. On the other hand, order evaluation data allow us to determine how much disorder can be tolerated in a sample before significant departures from the ideal sensing properties.

Different order evaluation methods are available. For most of them, the starting point of the analysis is a 2D image of the periodic structure obtained by optical microscopy, atomic force microscopy (AFM), scanning electron microscopy (SEM), or transmission electron microscopy (TEM). The main characteristics of the different order evaluation methods are summarized at the end of the chapter, in Table 2.

Table 2. Comparative summary of the different methods for order quantification of periodic patterns.

\begin{tabular}{cccc}
\hline Main Methods & Source & Information Provided & Main Fields of Application \\
\hline $\begin{array}{c}\text { 2D Fourier transform } \\
\text { (2DFT) [94-98] }\end{array}$ & $\begin{array}{c}\text { 2D image acquired by optical } \\
\text { microscopy, SEM, TEM, AFM }\end{array}$ & $\begin{array}{c}\text { Symmetry, periodicity } \\
\text { and orientation. } \\
\text { Quantification of degree of order }\end{array}$ & $\begin{array}{c}\text { Block-copolymer self-assembly, } \\
\text { breath figures, colloidal assembly }\end{array}$ \\
\hline $\begin{array}{c}\text { Pair correlation function } \\
\text { (PCF) [99-101] }\end{array}$ & $\begin{array}{c}\text { 2D image acquired by optical } \\
\text { microscopy, SEM, TEM, AFM }\end{array}$ & $\begin{array}{c}\text { Quantification of order by deviation } \\
\text { from the expected periodicity }\end{array}$ & $\begin{array}{c}\text { Block-copolymer self-assembly, } \\
\text { colloidal assembly }\end{array}$ \\
\hline Voronoi tessellation [102,103] & $\begin{array}{c}\text { 2D image acquired by optical } \\
\text { microscopy, SEM, TEM, AFM }\end{array}$ & $\begin{array}{c}\text { Identification and quantification of } \\
\text { defects. Quantification of order by } \\
\text { conformal entropy calculation }\end{array}$ & $\begin{array}{c}\text { Block-copolymer self-assembly, } \\
\text { breath figures, colloidal assembly }\end{array}$ \\
\hline Debye ring [104,105] & Real sample, possibility of \\
real-time monitoring & $\begin{array}{c}\text { Qualitative information about } \\
\text { periodicity and crystallite size. } \\
\text { Determination of the spacing. }\end{array}$ & Breath figures, colloidal assembly \\
\hline
\end{tabular}

\subsection{Order Quantification by Fourier Transform}

When dealing with 2D ordered structures, and specifically with hexagonally arranged patterns as those obtainable by self-assembly, Fourier transform can provide both qualitative and quantitative information. By providing information about the spatial frequencies 
of an image, in fact, the 2D Fourier transform (2DFT) is a useful tool for extracting from the original image evidence about the symmetry, periodicity and orientation of specific patterns. For this reason, it has been extensively used for analyzing the order of colloidal assemblies [94-98].

For a triangular lattice with a hexagonal spot arrangement, whether they be particles or pores, the first order peak of the 2DFT consists of six distinct spots on the edges of a hexagon (Figure 6(a-i)). When the arrangement is not perfectly hexagonal and defects are present, the 2DFT pattern turns into a ring or disc-shaped form (Figure 6(a-ii)) [96]. The presence of a narrow ring indicates a preserved short-distance periodicity of the network and slightly disturbed long-range order. As the ring gets larger, the periodicity is more and more disturbed. When the 2DFT pattern has a disc shape, the degree of order of the structure is very low.

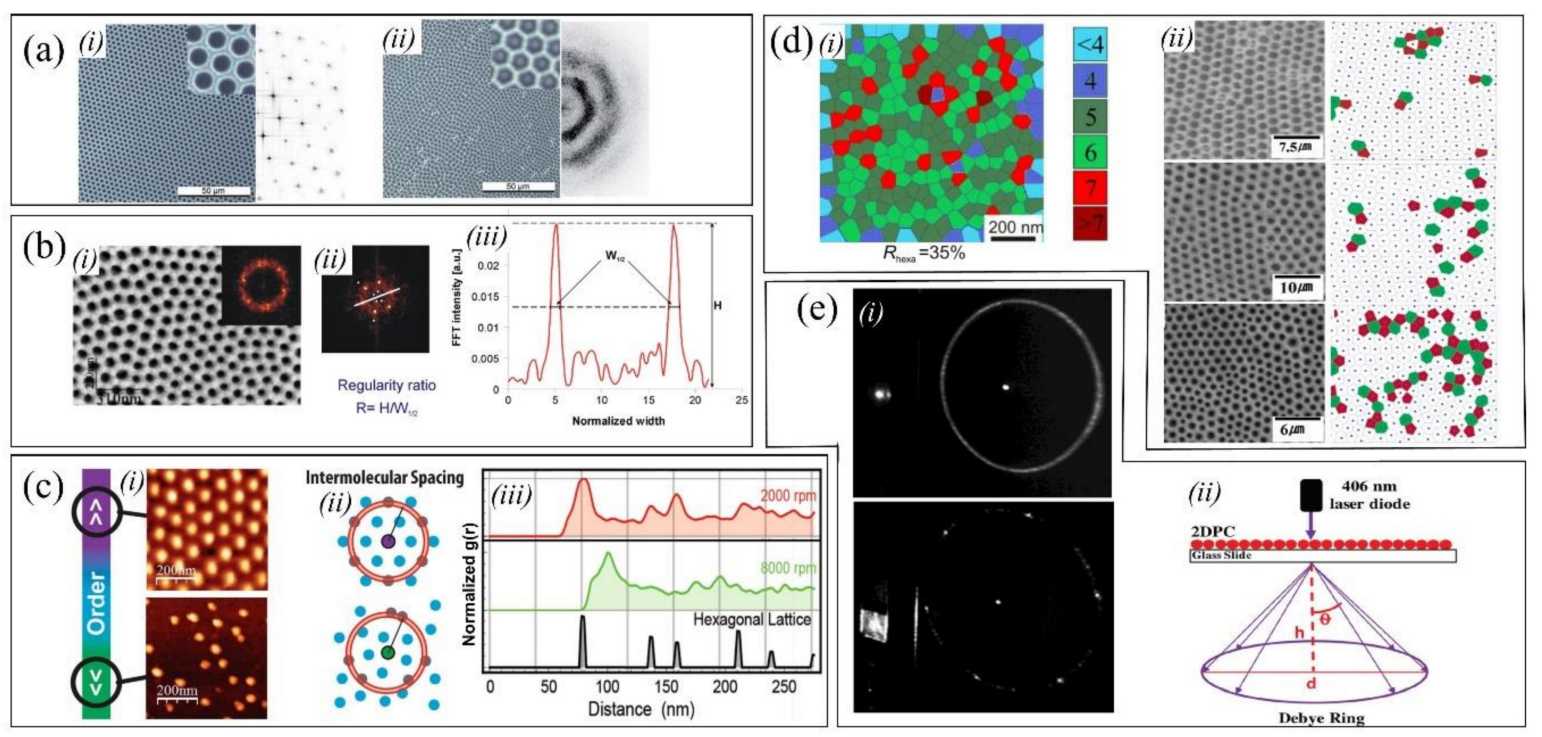

Figure 6. (a) Optical microscopy pictures of diblock copolymer honeycomb films obtained by breath figures with a blowup in the inset and corresponding 2D Fourier transforms. Reproduced from Ref. [96] with permission from the 2010 Royal Society of Chemistry. (b) FE-SEM top-view of anodic porous titania together with its 2D Fourier transform in the inset (i); profile analysis (iii) of the 2D Fourier transform intensity of a similar sample along the marked solid line in (ii). Reproduced from Ref. [97] with permission from 2010 Elsevier Ltd. (c) AFM micrographs of diblock copolymer reverse micelles of PS-b-P2VP assembled at different spin-coating speed (i) showing highly ordered organization (top) and very low density (bottom); graphical representation of pair correlation function of the corresponding AFM images (ii) and plotted values of pair correlation function (iii) of the highly ordered system (red), disordered system (green) and calculated hexagonal lattice (black). Reproduced from Ref. [99]. (d) Voronoi tessellation performed on SEM image of a block-copolymer thin film prepared by spin-coating with legend highlighting the correspondences between colors and number of the sides of the polygons and evaluation of the corresponding degree of hexagonal order (i); SEM images and corresponding Voronoi polygons of breath figure patterns of $C A B$ prepared by different methods: pores with five- and seven-nearest neighbors are colored red and green, respectively (ii). Reproduced from Ref. [100] with permission from the 2014 Royal Society of Chemistry and from Ref. [101] with permission from the 2004 American Chemical Society, respectively. (e) Diffraction of normally incident laser beam transmitted through a uniform hexagonal latex monolayer using a $4 \mathrm{~mm}$ diameter beam spot (top) and reducing the beam spot to $0.25 \mathrm{~mm}$ (bottom) which changes the diffraction pattern to six symmetrical spots (i); graphical representation of Debye ring obtained using a violet laser pointer ( $406 \mathrm{~nm}, 2.2 \mathrm{~mm}$ spot size) to illuminate the 2DPC at normal incidence (ii). Reproduced from Ref. [102] with permission from the 2004 American Chemical Society and from Ref. [103] with permission from the 2017 Royal Society of Chemistry, respectively.

Besides such a qualitative assessment of the hexagonal order, 2DFT can also provide a quantification of the order level. In highly ordered 2D structures, in fact, the reciprocal of the distance of the six distinct spots from the center of the 2DFT provides the periodicity of the structure in the real image. The profile analysis along the center and two opposite distinct 
points of the 2DFT should show two very smooth and high peaks. Sulka et al. exploited this characteristic to quantify the regularity of pore arrangement in $\mathrm{TiO}_{2}$ nanoporous surfaces [97]. To do so, they defined a regularity factor (R) as the ratio of the peak height in the 2DFT profile to the width of the peak at the half-maximum (Figure 6b). Because the nanoporous structure analyzed could have different interpore distances, the same image area could include a different number of pores, which would affect the peak intensity in the 2DFT profile. To overcome the influence of pore numbers on the analysis, the average regularity factor calculated from the 2DFT profiles was multiplied by pore density (n). By following this procedure, the authors could compare the regularity of samples fabricated by applying different potentials in the electrodeposition of $\mathrm{TiO}_{2}$ nanoporous structure.

\subsection{Order Quantification by Pair Correlation Function}

Another method for quantifying the order of 2D periodic structures is based on the pair correlation function (PCF). The PCF is typically used to describe quantitatively the internal structure of fluids and quantifies how the particle of interest is surrounded by other particles. For example, for a rarefied gas in which there is no internal structure, the PCF has a constant value for all radial distances. In contrast, for a solid system in which molecules are almost regularly located, the PCF has sharply peaked values at the positions of particles, while is nearly zero at the positions where particles are seldom located. Therefore, the PCF provides a useful method to investigate the degree of order of a self-assembled structure [104].

The PCF is expressed by $\mathrm{g}(\mathrm{r})$, and it describes the probability of finding a particle at a distance $r$ from a given particle in the 2D space. To calculate it, an initial central particle $a$ is chosen as the origin. A circular shell of width $d r$ expands from the center to a radius distance $r$. Any particles inside a circular shell of width $\Delta r$ are counted together and binned to produce the neighbor probability $n_{n}(r)$ as a function of distance, weighted by the particle density, $\rho$ :

$$
g(r)=\frac{n_{n}(r)}{2 \pi r \Delta r \rho}
$$

A schematic representation of $\mathrm{g}(\mathrm{r})$ is shown in Figure 6(c-ii). The particle coordinates must be determined by analyzing a microscopy image (SEM, TEM, AFM) of the periodic structure with an image analysis software such as ImageJ [105]. A practical example of the application of the PCF to colloidal patterns is provided in a recent article by Bumstead et al. [99]. In this paper, the authors developed a mathematical method, named disLocate, to rapidly assess and quantify the two-dimensional order of PS-b-P2VP diblock copolymer micelles assembled by spin-coating at different speeds. When the AFM image shows that micelles are organized in a hexagonally ordered pattern, as in the top frame of Figure 6(c-i), the corresponding PCF finds particles at regular intervals as the distance from the central particle increases, as schematized by Figure 6(c-ii) top, and plotted in Figure 6(c-iii) top. These intervals are specific for the expected geometry and can therefore be predicted by calculation. For instance, for the hexagonal pattern, if $d_{p}$ is the particle diameter, the positions of these peaks correspond to $d_{p}, \sqrt{3} d_{p}, 2 d_{p}, \sqrt{7} d_{p}, 3 d_{p}, 2 \sqrt{3} d_{p}, \sqrt{13} d_{p}, 4 d_{p}$ etc, represented by the bottom plot of Figure 6(c-iii). As the periodicity of the pattern is weakened or lost, like in the bottom case of Figure $6 c$, the main $g(r)$ peaks get further from these predicted positions.

\subsection{Order Quantification by Voronoi Tessellation}

A simple and intuitive method for evaluating the degree of order in 2D periodic structures is the one based on Voronoi tessellation. A Voronoi polygon is the smallest convex polygon that surrounds a point so that its sides are perpendicularly bisecting the lines connecting the central point to the ones immediately neighbors. Because every point belonging to a polygon is closer to the center point of that polygon than to every other center points, this technique is commonly used in geographical or networking applications, for example, to locate the closest airport to one position on a map or the closest cell tower 
to which a mobile phone will connect during a call. On a far more reduced scale, on the other hand, Voronoi tessellation can be used to identify lattice defects. Because Voronoi polygons have a number of sides equal to the number of nearest neighbors of the point in the center, they allow the quantification of the deviation from the expected geometry. This can be intuitively understood by the observation of the diagrams in Figure 6(d-i), showing a Voronoi analysis applied to $1 \mu \mathrm{m} \times 1 \mu \mathrm{m}$ FE-SEM images of a BCP thin film prepared by spin-coating [100]. If a periodic structure is expected, hexagonal arrangement, in this case, a perfect honeycomb tiling should be observed, with each cell consisting of a regular hexagon. In the example, each different polygon is filled by a different color, and only $35 \%$ of them are hexagons, indicated by light green. For a square arrangement, on the other hand, the Voronoi diagram consists of square cells, here filled by dark blue.

When studying the order of quasi-periodic structures, any deviation from the ideal tessellation can be interpreted as degradation of order. As such, these tools can be used for order quantification. Usually, in Voronoi analysis, order quantification is conducted in terms of conformation entropy $(S)$ of the pattern [101], where the term entropy is perhaps improperly used to indicate the degree of disorder. It is calculated as

$$
S=-\Sigma P_{n} \ln P_{n}
$$

where $n$ is the number of sides of the Voronoi polygon (the coordination number), and $P_{n}$, the fraction of polygons with $n$. The conformational entropy of the quasi-periodic structure is then compared with the reference value $S=1.71$, obtained in the case of a totally random distribution of points, and with the null value, ideally obtainable with an array of infinite dimensions and perfectly ordered. As an example of this approach, Figure 6(d-ii) reports SEM images and corresponding Voronoi tessellation of microporous film obtained by breath figure templating. The aim of the authors was to compare different processing conditions to obtain cellulose acetate butyrate ( $\mathrm{CAB}$ ) microporous films: from chloroform solution (top), from THF solution (middle), and from THF by spin-coating (bottom). As in the majority of the self-assembly methods, the expected geometry for breath figure films is the hexagonal one. By using chloroform as a solvent, the probability of having five, six, or seven prime neighbors is respectively $P 5=0.061, P 6=0.893$ and $P 7=0.046$, which gives conformational entropy $S=0.41$, far from the value 1.71 calculated for a completely disordered distribution. For the THF case, $S$ values are slightly increased to 0.48 , while for the breath figure film obtained by spin-coating a THF solution, $S$ becomes 0.86 , indicating that more defects in hexagonal packing are generated by these conditions. This trend is immediately evidenced by the increasing number of colored polygons, indicating packing defects.

\subsection{Laser Diffraction}

Sometimes, a fast and qualitative method for order evaluation is necessary. This is especially desirable during the process optimization attempts, to have a fast response of the modification made to the process, as well as during the preparation of a series of samples, to have quick quality control. In this view, laser diffraction can help to recognize immediately whether the obtained pattern is completely disordered or it possesses some periodicity.

The technique is suitable for studying 2D distributions of objects (particles or pores) on a transparent substrate, and consists of illuminating the sample at normal incidence with a laser beam, so that the diffracted light is projected to a screen on the other side of the sample. As shown in Figure 6(e-i), when the sample is hit by the laser beam a ring is generated on the screen, which becomes six symmetrical spots within a diffuse ring when the irradiated portion of the sample is a perfect hexagonal array, and the laser spot is smaller than the illuminated crystallite [102]. These images are called Debye rings and are 2D optical analogues of the common X-ray diffraction from 3D samples of crystalline powders (Debye-Scherrer method).

Laser diffraction is very useful, for example, to evaluate the order of 2DPCs [103]. In fact, by monitoring the Debye ring diffraction we can quantitatively determine the 
2DPC ordering without perturbing the sample and without using a microscope. A laser pointer is normally sufficient to analyzing this kind of samples, obtaining a quick comeback on what is going on. As 2DPCs are often obtained by assembling microspheres at the air/water interface, it is even possible to analyze the Debye ring directly on the monolayer colloidal crystal floating on the water surface. This kind of monitoring can be particularly advantageous by the moment that, once the 2DPC is transferred from water to a substrate, the self-assembly process is frozen and it is practically impossible to modify it.

Besides the qualitative information about periodicity and crystallite size, laser diffraction is able to provide also information about the spacing. As depicted in Figure 6(e-ii), the diameter of the Debye ring projected onto the screen in fact depends on both the 2DPC particle spacing, $a$ and the distance, $h$ between the 2DPC and the screen, according to the following formulas:

$$
\begin{aligned}
& \theta=\tan ^{-1} \frac{d}{2 h} \\
& a=\frac{2 \lambda}{\sqrt{3} \sin \theta}
\end{aligned}
$$

Hence, by measuring the ring diameter and its distance from the sample (knowing the wavelength of the laser, $\lambda$ ), the particle spacing can be calculated. When the parameters of the Debye ring are used to calculate the spacing of a 2DPC on the water surface, one should take into account that the diffraction of the 2DPC is shifted due to refraction of the light by the air-water interfaces. Consequently, the Debye ring diameter of a 2DPC on the water surface will be smaller than that from an identical 2DPC on a glass slide. Anyway, this deviation is related to the thickness of the water layer and can be calculated [103].

\section{Conclusions}

Self-assembly processes represent a cost-effective option for the mass production of technologically advanced platforms based on OFs. The nanofabrication of highly ordered structures on a non-conventional substrate like the OF is challenging, whilst coupling the $\mathrm{OF}$ with ordered nanopatterns is required for the development of several advanced sensing and biosensing technological platforms. Bottom-up approaches are capable of building nanostructures, hierarchically ordered structures and sophisticated micro- and nanopatterns on the OFs with a high degree of order and a wide geometrical parameters control, according to the characteristics summarized in Table 1. At the same time, they have the advantage of high throughput and low-cost processing, two key elements suitable to accelerate the real market exploitation of lab-on-fiber probes.

Withstanding this, a few limitations characteristic of these self-assembling approaches still hinder the establishment of these technologies as the springboard for the transition from valuable lab-on-fiber proofs of concept toward the market.

One of the main constraints relies on the limited number of implementable geometries. The self-organization process at the basis of the pattern formation typically consists of creating the controlled conditions suitable to push the "disassembled" system toward an equilibrium state corresponding to the self-packing of nanostructures in a compact fashion. When operating with nanospheres the natural achievement of this minimum energy equilibrium state corresponds to hexagonal packing in a 2D planar pattern as well as fcc symmetry in 3D structures. Most of the reported structures feature indeed similar patterns directly or indirectly obtained by such basic geometries.

Currently, remarkable attempts to enlarge the set of attainable geometries are only associated with the DSA method, a combination of top-down and bottom-up approaches, which using substrates patterned with linear, hexagonal and circular structures, demonstrated the formation of unusual patterns. Nonetheless, nature already demonstrated that different geometries are surely possible in full self-assembly approaches. Self-assembling of aperiodically ordered structures is present in nature too but controlled methods to artificially build photonic quasicrystals by auto-organization are still not demonstrated. 
The enlargement of the attainable structures is important for conferring versatility to the nanofabrication process. Overall, hierarchical structures, DSA and post-processing treatments can open new routes to self-assembling nanostructures.

Nanofabrication techniques based on self-assembly processes have already proved their usefulness in the creation of OF sensors and biosensors, but many self-assembly processes are still unexplored with OFs [4]. So far, the assembly of colloidal crystals has surely given the most consistent contribution, especially because it can count on a consolidated assembly process and on the post-assembly modification processes provided by NSL. Hierarchical structures based on nanospheres with different diameters [106-109], as well as on the assembly of anisotropic particles [110], could offer new degrees of freedom in pattern design and open new opportunities related to the NSL development, as shown by the examples reported in Figure 7.
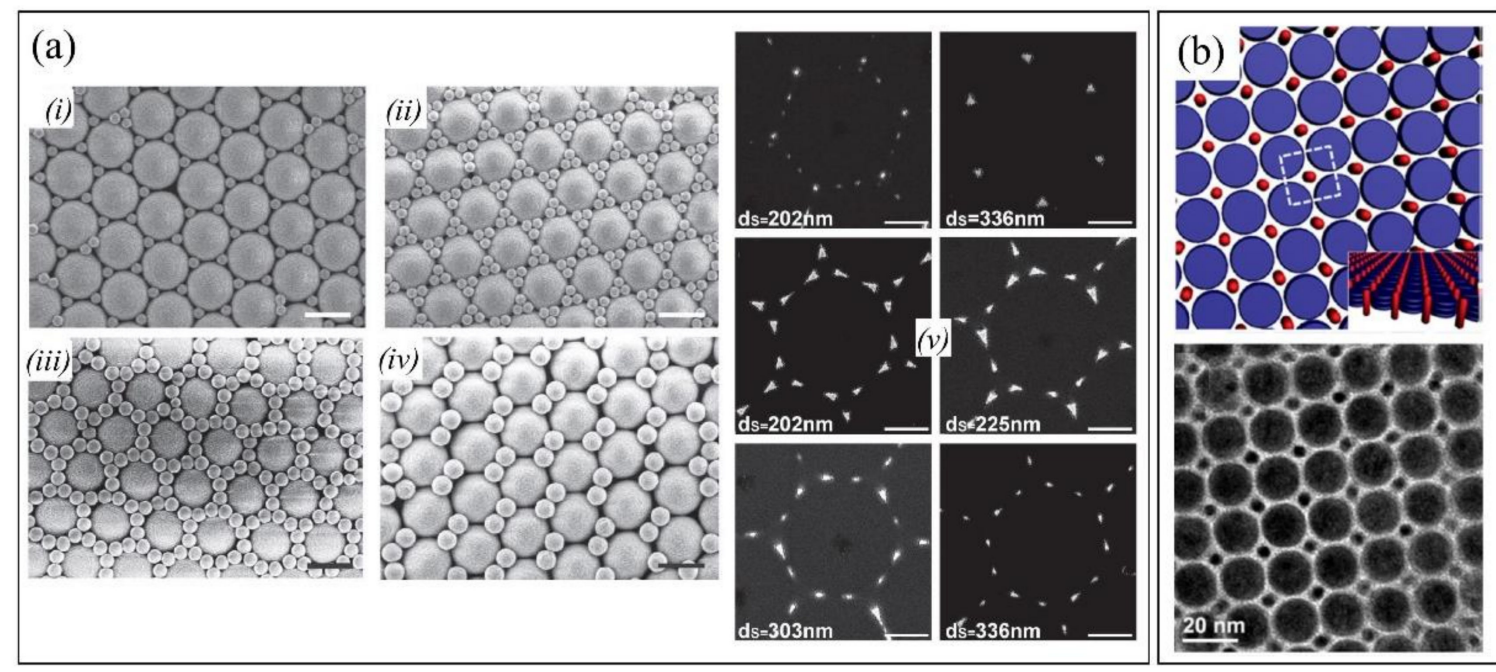

Figure 7. (a) SEM micrographs of different binary assemblies obtained by adjusting the size ratio $\mathrm{R}_{\mathrm{S}}$ and the number ratio $\mathrm{R}_{\mathrm{N}}$ between the small and the large particles (i-iv), and possible metal nanotriangle arrays obtainable by NSL with binary assemblies (v). Scale bars are $1 \mu \mathrm{m}$ for (i-iv) and $400 \mathrm{~nm}$ for (v). Reproduced from Ref. [109] with permission from 2011 WILEY-VCH Verlag GmbH and Co. KGaA, Weinheim, Germany. (b) Structural model of AB-type binary superlattice (top) and corresponding binary superlattice self-assembled from $13.2 \mathrm{~nm} \mathrm{LaF}$ nanodisks and CdSe/CdS nanorods (bottom). Reproduced from Ref. [110] with permission from the 2015 American Chemical Society.

A less exploited and therefore potentially capable of opening new scenarios is also the breath figure patterning. Great potentiality is promised by the DSA paradigm, which combines BCP self-assembly with patterns obtained by classical lithographic techniques, which surprisingly has only been marginally explored on OFs so far.

Another key limitation in self-assembly approaches relies on defect formation, which seems inevitable for all proposed techniques at least in the "long-range" scale. Postprocessing treatments can be used to reduce the number of defects and increase the order but maybe it is equally important to define how much defectivity can be tolerated by the system, more than aiming at perfect ordered patterns. Periodic order is important in establishing photonic resonances (i.e., guided resonances) and plasmonic resonances (exploited in SPR and SERS probes). A "strong" deviation from the order can surely affect the formation of such resonances, but a "weak" deviation can only slightly change their spectral characteristics (i.e., slight spectral shifting or decreasing of the resonance Q-factor can be expected). Several order quantification methods are available both for batch-tobatch quality control and for an immediate evaluation of the self-assembly process, as summarized in Table 2. For example, an order evaluation tool like the Debye ring can be applied directly to the sample while it is forming or immediately after its preparation, without damaging it, and therefore can be used in the optimization steps to find the 
conditions that allow obtaining the most ordered structures which are achievable by the technique employed.

Another important aspect related to periodic order (and related defects) pertains to reproducibility and uniformity. Ordered structures are in general more effective than the random ones both in terms of reproducibility and of efficiency of the photonic response. When the photonic structures are created by NSL and the outcome is SERS detection, for example, the density of plasmonic structures strongly affects the SERS activity of the substrate since, by increasing it, an increment in the number of hot spots is produced. In addition, the periodicity of ordered nanostructures leads to a uniform distribution of hot spots over the sample. In turn, the deviation from the order affects the possibility to get repeatable quantitative measurements.

A systematic evaluation of the degree of order is often missing whilst the assessment of the degree of order is an important best practice useful to push the proposed self-assembly methods to a mature technological level.

Definitively, nanofabrication of highly ordered nanostructures by bottom-up methods demonstrated great potentialities in terms of reproducibility and repeatability for OF probes development. Even if each technique presents some limitations or a reduced versatility with respect to classical nanofabrication tools, the described self-assembly methods collectively represent an alternative tool to integrate advanced materials with the OF in order to attain advanced lab-on-fiber devices. The growth margins of the self-assembly processes are still enormous. Further research efforts are required to overcome current limitations and fully exploit the potentialities through the rigorous assessment of the fabrication processes.

Author Contributions: M.P. and F.G.: Writing—original draft, review \& editing. All authors have read and agreed to the published version of the manuscript.

Funding: This research received no external funding.

Institutional Review Board Statement: Not applicable.

Informed Consent Statement: Not applicable.

Data Availability Statement: Not applicable.

Conflicts of Interest: The authors declare no conflict of interest.

\section{References}

1. Martin, J.S.; Smith, N.A.; Francis, C.D. Removing the entropy from the definition of entropy: Clarifying the relationship between evolution, entropy, and the second law of thermodynamics. Evol. Educ. Outreach 2013, 6, 30. [CrossRef]

2. Ricciardi, A.; Crescitelli, A.; Vaiano, P.; Quero, G.; Consales, M.; Pisco, M.; Esposito, E.; Cusano, A. Lab-on-fiber technology: A new vision for chemical and biological sensing. Analyst 2015, 140, 8068-8079. [CrossRef] [PubMed]

3. Vaiano, P.; Carotenuto, B.; Pisco, M.; Ricciardi, A.; Quero, G.; Consales, M.; Crescitelli, A.; Esposito, E.; Cusano, A. Lab on Fiber Technology for biological sensing applications. Laser Photonics Rev. 2016, 10, 922-961. [CrossRef]

4. Galeotti, F.; Pisco, M.; Cusano, A. Self-assembly on optical fibers: A powerful nanofabrication tool for next generation "lab-onfiber" optrodes. Nanoscale 2018, 10, 22673-22700. [CrossRef]

5. Joannopoulos, J.D.; Johnson, S.G.; Winn, J.N.; Meade, R.D. Photonic Crystals: Molding the Flow of Light, 2nd ed.; Princeton University Press: Princeton, NJ, USA, 2008; pp. 1-286.

6. Lin, S.Y.; Chow, E.; Hietala, V.; Villeneuve, P.R.; Joannopoulos, J.D. Experimental demonstration of guiding and bending of electromagnetic waves in a photonic crystal. Science 1998, 282, 274-276. [CrossRef]

7. Chow, E.; Lin, S.Y.; Johnson, S.G.; Villeneuve, P.R.; Joannopoulos, J.D.; Wendt, J.R.; Vawter, G.A.; Zubrzycki, W.; Hou, H.; Alleman, A. Three-dimensional control of light in a two-dimensional photonic crystal slab. Nature 2000, 407, 983-986. [CrossRef]

8. Valanju, P.M.; Walser, R.M.; Valanju, A.P. Wave refraction in negative-index media: Always positive and very inhomogeneous. Phys. Rev. Lett. 2002, 88. [CrossRef]

9. Noda, S.; Chutinan, A.; Imada, M. Trapping and emission of photons by a single defect in a photonic bandgap structure. Nature 2000, 407, 608-610. [CrossRef]

10. Knight, J.C.; Broeng, J.; Birks, T.A.; Russel, P.S.J. Photonic band cap guidance in optical fibers. Science 1998, 282, 1476-1478. [CrossRef]

11. Russell, P. Photonic crystal fibers. Science 2003, 299, 358-362. [CrossRef]

12. Pitruzzello, G.; Krauss, T.F. Photonic crystal resonances for sensing and imaging. J. Opt. 2018, 20. [CrossRef] 
13. Macia, E. The role of aperiodic order in science and technology. Rep. Prog. Phys. 2006, 69, 397-441. [CrossRef]

14. Press Release. NobelPrize.org. Nobel Media AB 2021. Available online: https://www.nobelprize.org/prizes/chemistry/2011 /press-release/ (accessed on 11 February 2021).

15. Shechtman, D.; Blech, I.; Gratias, D.; Cahn, J.W. Metallic Phase with Long-Range Orientational Order and No Translational Symmetry. Phys. Rev. Lett. 1984, 53, 1951-1953. [CrossRef]

16. Bendersky, L. Quasicrystal with One-Dimensional Translational Symmetry and a Tenfold Rotation Axis. Phys. Rev. Lett. 1985, 55, 1461-1463. [CrossRef]

17. Bancel, P.A.; Heiney, P.A. Icosahedral Aluminum Transition-Metal Alloys. Phys. Rev. B 1986, 33, 7917-7922. [CrossRef]

18. Wang, N.; Chen, H.; Kuo, K.H. Two-Dimensional Quasi-Crystal with Eightfold Rotational Symmetry. Phys. Rev. Lett. 1987, 59, 1010-1013. [CrossRef]

19. Ishimasa, T.; Nissen, H.U.; Fukano, Y. New Ordered State between Crystalline and Amorphous in Ni-Cr Particles. Phys. Rev. Lett. 1985, 55, 511-513. [CrossRef]

20. Fischer, S.; Exner, A.; Zielske, K.; Perlich, J.; Deloudi, S.; Steurer, W.; Lindner, P.; Foerster, S. Colloidal quasicrystals with 12-fold and 18-fold diffraction symmetry. Proc. Natl. Acad. Sci. USA 2011, 108, 1810-1814. [CrossRef]

21. Zeng, X.B.; Ungar, G.; Liu, Y.S.; Percec, V.; Dulcey, S.E.; Hobbs, J.K. Supramolecular dendritic liquid quasicrystals. Nature 2004, 428, 157-160. [CrossRef]

22. Hayashida, K.; Dotera, T.; Takano, A.; Matsushita, Y. Polymeric quasicrystal: Mesoscopic quasicrystalline tiling in ABC star polymers. Phys. Rev. Lett. 2007, 98. [CrossRef]

23. Talapin, D.V.; Shevchenko, E.V.; Bodnarchuk, M.I.; Ye, X.; Chen, J.; Murray, C.B. Quasicrystalline order in self-assembled binary nanoparticle superlattices. Nature 2009, 461, 964-967. [CrossRef] [PubMed]

24. Mikhael, J.; Roth, J.; Helden, L.; Bechinger, C. Archimedean-like tiling on decagonal quasicrystalline surfaces. Nature 2008, 454, 501-504. [CrossRef]

25. Vardeny, Z.V.; Nahata, A.; Agrawal, A. Optics of photonic quasicrystals. Nat. Photonics 2013, 7, 177-187. [CrossRef]

26. Chan, Y.S.; Chan, C.T.; Liu, Z.Y. Photonic band gaps in two dimensional photonic quasicrystals. Phys. Rev. Lett. 1998, 80, 956-959. [CrossRef]

27. Kaliteevski, M.A.; Brand, S.; Abram, R.A.; Krauss, T.F.; DeLa Rue, R.; Millar, P. Two-dimensional Penrose-tiled photonic quasicrystals: From diffraction pattern to band structure. Nanotechnology 2000, 11, 274-280. [CrossRef]

28. Dal Negro, L.; Oton, C.J.; Gaburro, Z.; Pavesi, L.; Johnson, P.; Lagendijk, A.; Righini, R.; Colocci, M.; Wiersma, D.S. Light transport through the band-edge states of Fibonacci quasicrystals. Phys. Rev. Lett. 2003, 90. [CrossRef]

29. Della Villa, A.; Enoch, S.; Tayeb, G.; Pierro, V.; Galdi, V.; Capolino, F. Band gap formation and multiple scattering in photonic quasicrystals with a Penrose-type lattice. Phys. Rev. Lett. 2005, 94. [CrossRef]

30. Ricciardi, A.; Gallina, I.; Campopiano, S.; Castaldi, G.; Pisco, M.; Galdi, V.; Cusano, A. Guided resonances in photonic quasicrystals. Opt. Exp. 2009, 17, 6335-6346. [CrossRef]

31. Gallina, I.; Pisco, M.; Ricciardi, A.; Campopiano, S.; Castaldi, G.; Cusano, A.; Galdi, V. Guided resonances in photonic crystals with point-defected aperiodically-ordered supercells. Opt. Exp. 2009, 17, 19586-19598. [CrossRef]

32. Pisco, M.; Ricciardi, A.; Gallina, I.; Castaldi, G.; Campopiano, S.; Cutolo, A.; Cusano, A.; Galdi, V. Tuning efficiency and sensitivity of guided resonances in photonic crystals and quasi-crystals: A comparative study. Opt. Exp. 2010, 18, 17280-17293. [CrossRef] [PubMed]

33. Ricciardi, A.; Pisco, M.; Gallina, I.; Campopiano, S.; Galdi, V.; O’Faolain, L.; Krauss, T.F.; Cusano, A. Experimental evidence of guided-resonances in photonic crystals with aperiodically ordered supercells. Opt. Lett. 2010, 35, 3946-3948. [CrossRef] [PubMed]

34. Ricciardi, A.; Pisco, M.; Cutolo, A.; Cusano, A.; Faolain, L.O.; Krauss, T.F.; Castaldi, G.; Galdi, V. Evidence of guided resonances in photonic quasicrystal slabs. Phys. Rev. B 2011, 84. [CrossRef]

35. Notomi, M.; Suzuki, H.; Tamamura, T.; Edagawa, K. Lasing action due to the two-dimensional quasiperiodicity of photonic quasicrystals with a Penrose lattice. Phys. Rev. Lett. 2004, 92. [CrossRef] [PubMed]

36. Mahler, L.; Tredicucci, A.; Beltram, F.; Walther, C.; Faist, J.; Beere, H.E.; Ritchie, D.A.; Wiersma, D.S. Quasi-periodic distributed feedback laser. Nat. Photonics 2010, 4, 165-169. [CrossRef]

37. Boriskina, S.V.; Lee, S.Y.K.; Amsden, J.J.; Omenetto, F.G.; Dal Negro, L. Formation of colorimetric fingerprints on nano-patterned deterministic aperiodic surfaces. Opt. Exp. 2010, 18, 14568-14576. [CrossRef]

38. Lee, S.Y.; Amsden, J.J.; Boriskina, S.V.; Gopinath, A.; Mitropolous, A.; Kaplan, D.L.; Omenetto, F.G.; Dal Negro, L. Spatial and spectral detection of protein monolayers with deterministic aperiodic arrays of metal nanoparticles. Proc. Natl. Acad. Sci. USA 2010, 107, 12086-12090. [CrossRef]

39. Mokkapati, S.; Catchpole, K.R. Nanophotonic light trapping in solar cells. J. Appl. Phys. 2012, 112. [CrossRef]

40. Benagli, S.; Borrello, D.; Vallat-Sauvain, E.; Meier, J.; Kroll, U.; Hoetzel, H.; Bailat, J.; Steinhauser, J.; Marmelo, M.; Monteduro, G.; et al. High-Efficiency Amorphous Silicon Devices on LPCVD-ZnO TCO Prepared in Industrial KAI-M Reactor. In Proceedings of the 24th European Photovoltaic Solar Energy Conference, Hamburg, Germany, 21-25 September 2009; pp. 2293-2298.

41. Battaglia, C.; Hsu, C.-M.; Söderström, K.; Escarré, J.; Haug, F.-J.; Charrière, M.; Boccard, M.; Despeisse, M.; Alexander, D.T.L.; Cantoni, M.; et al. Light Trapping in Solar Cells: Can Periodic Beat Random? ACS Nano 2012, 6, 2790-2797. [CrossRef] 
42. Micco, A.; Ricciardi, A.; Pisco, M.; La Ferrara, V.; Mercaldo, L.V.; Veneri, P.D.; Cutolo, A.; Cusano, A. Light trapping efficiency of periodic and quasiperiodic back-reflectors for thin film solar cells: A comparative study. J. Appl. Phys. 2013, 114. [CrossRef]

43. Li, J.; Herman, P.R.; Valdivia, C.E.; Kitaev, V.; Ozin, G.A. Colloidal photonic crystal cladded optical fibers: Towards a new type of photonic band gap fiber. Opt. Exp. 2005, 13, 6454-6459. [CrossRef]

44. Lin, Y.; Herman, P.R.; Xu, W. In-fiber colloidal photonic crystals and the formed stop band in fiber longitudinal direction. J. Appl. Phys. 2007, 102, 073106. [CrossRef]

45. Yan, H.; Wang, M.; Ge, Y.; Yu, P. Colloidal crystals self-assembled on the end face of fiber: Fabrication and characterizations. Opt. Fiber Technol. 2009, 15, 324-327. [CrossRef]

46. Sansone, L.; Macchia, E.; Taddei, C.; Torsi, L.; Giordano, M. Label-free optical biosensing at femtomolar detection limit. Sens. Actuators B Chem. 2018, 255, 1097-1104. [CrossRef]

47. Moon, J.H.; Yi, G.-R.; Yang, S.-M. Fabrication of hollow colloidal crystal cylinders and their inverted polymeric replicas. J. Colloid Interf. Sci. 2005, 287, 173-177. [CrossRef]

48. Haibin, N.; Ming, W.; Wei, C. Sol-gel co-assembly of hollow cylindrical inverse opals and inverse opal columns. Opt. Exp. 2011, 19, 25900-25910. [CrossRef]

49. Hatton, B.; Mishchenko, L.; Davis, S.; Sandhage, K.H.; Aizenberg, J. Assembly of large-area, highly ordered, crack-free inverse opal films. Proc. Natl. Acad. Sci. USA 2010, 107, 10354. [CrossRef]

50. Ni, H.; Wang, M.; Li, L.; Chen, W.; Wang, T. Photonic-Crystal-Based Optical Fiber Bundles and Their Applications. IEEE Photonics J. 2013, 5, 2400213. [CrossRef]

51. Guo, Y.; Chen, L.; Zhu, J.; Ni, H.; Xia, W.; Wang, M. Compact 3D photonic crystals sensing platform with 45 degree angle polished fibers. Opt. Fiber Technol. 2017, 36, 187-194. [CrossRef]

52. Pisco, M.; Galeotti, F.; Quero, G.; Grisci, G.; Micco, A.; Mercaldo, L.V.; Veneri, P.D.; Cutolo, A.; Cusano, A. Nanosphere lithography for optical fiber tip nanoprobes. Light Sci. Appl. 2017, 6. [CrossRef]

53. Quero, G.; Zito, G.; Manago, S.; Galeotti, F.; Pisco, M.; De Luca, A.C.; Cusano, A. Nanosphere Lithography on Fiber: Towards Engineered Lab-On-Fiber SERS Optrodes. Sensors 2018, 18, 680. [CrossRef]

54. Managò, S.; Quero, G.; Zito, G.; Tullii, G.; Galeotti, F.; Pisco, M.; De Luca, A.C.; Cusano, A. Tailoring lab-on-fiber SERS optrodes towards biological targets of different sizes. Sens. Actuators B Chem. 2020, 129321. [CrossRef]

55. Chang, S.-H.; Nyagilo, J.; Wu, J.; Hao, Y.; Davé, D.P. Optical Fiber-Based Surface-Enhanced Raman Scattering Sensor Using Au Nanovoid Arrays. Plasmonics 2012, 7, 501-508. [CrossRef]

56. Antohe, I.; Spasic, D.; Delport, F.; Li, J.; Lammertyn, J. Nanoscale patterning of gold-coated optical fibers for improved plasmonic sensing. Nanotechnology 2017, 28, 215301. [CrossRef]

57. Xia, M.; Zhang, P.; Leung, C.; Xie, Y.-H. SERS optical fiber probe with plasmonic end-facet. J. Raman Spectrosc. 2017, 48, 211-216. [CrossRef]

58. Liang, Y.; Yu, Z.; Li, L.; Xu, T. A self-assembled plasmonic optical fiber nanoprobe for label-free biosensing. Sci. Rep. 2019, 9, 7379. [CrossRef]

59. Liu, Y.; Guang, J.; Liu, C.; Bi, S.; Liu, Q.; Li, P.; Zhang, N.; Chen, S.; Yuan, H.; Zhou, D.; et al. Simple and Low-Cost Plasmonic Fiber-Optic Probe as SERS and Biosensing Platform. Adv. Opt. Mater. 2019, 7, 1900337. [CrossRef]

60. Liu, Y.; Zhang, N.; Li, P.; Bi, S.; Zhang, X.; Chen, S.; Peng, W. Nanopatterned evanescent-field fiber-optic interferometer as a versatile platform for gas sensing. Sens. Actuators B Chem. 2019, 301, 127136. [CrossRef]

61. Aitken, J. Breath Figures. Nature 1913, 90, 619-621. [CrossRef]

62. Zhang, A.; Bai, H.; Li, L. Breath Figure: A Nature-Inspired Preparation Method for Ordered Porous Films. Chem. Rev. 2015, 115, 9801-9868. [CrossRef] [PubMed]

63. Bui, V.-T.; Ko, S.H.; Choi, H.-S. Large-Scale Fabrication of Commercially Available, Nonpolar Linear Polymer Film with a Highly Ordered Honeycomb Pattern. ACS Appl. Mater. Interf. 2015, 7, 10541-10547. [CrossRef]

64. Tullii, G.; Donini, S.; Bossio, C.; Lodola, F.; Pasini, M.; Parisini, E.; Galeotti, F.; Antognazza, M.R. Micro- and Nanopatterned Silk Substrates for Antifouling Applications. ACS Appl. Mater. Interf. 2020, 12, 5437-5446. [CrossRef]

65. Galeotti, F.; Hartmann, L.; Botta, C. Robust surface patterning by parylene-reinforced breath figures: An enabling tool for liquid crystal microcell arrays. J. Colloid Interf. Sci. 2016, 465, 47-53. [CrossRef]

66. Galeotti, F.; Trespidi, F.; Pasini, M. Breath Figure-Assisted Fabrication of Nanostructured Coating on Silicon Surface and Evaluation of Its Antireflection Power. J. Nanomater. 2016, 2016, 3502310. [CrossRef]

67. Galeotti, F.; Mróz, W.; Scavia, G.; Botta, C. Microlens arrays for light extraction enhancement in organic light-emitting diodes: A facile approach. Org. Electron. 2013, 14, 212-218. [CrossRef]

68. Galeotti, F.; Chiusa, I.; Morello, L.; Gianì, S.; Breviario, D.; Hatz, S.; Damin, F.; Chiari, M.; Bolognesi, A. Breath figures-mediated microprinting allows for versatile applications in molecular biology. Eur. Polym. J. 2009, 45, 3027-3034. [CrossRef]

69. Galeotti, F.; Kozma, E.; Mróz, W.; Kutrzeba-Kotowska, B. Single-step shaping of fluorescent polymer beads by a reverse breath figure approach. RSC Adv. 2015, 5, 36315-36319. [CrossRef]

70. Leone, G.; Giovanella, U.; Bertini, F.; Hoseinkhani, S.; Porzio, W.; Ricci, G.; Botta, C.; Galeotti, F. Hierarchically structured, blue-emitting polymer hybrids through surface-initiated nitroxide-mediated polymerization and water templated assembly. J. Mater. Chem. C 2013, 1, 6585-6593. [CrossRef] 
71. Galeotti, F.; Trespidi, F.; Timò, G.; Pasini, M. Broadband and Crack-Free Antireflection Coatings by Self-Assembled Moth Eye Patterns. ACS Appl. Mater. Interf. 2014, 6, 5827-5834. [CrossRef]

72. Colombo, R.N.P.; Petri, D.F.S.; Córdoba de Torresi, S.I.; Gonçales, V.R. Porous Polymeric Templates on ITO Prepared by Breath Figure Method for Gold Electrodeposition. Electrochim. Acta 2015, 158, 187-195. [CrossRef]

73. Kon, K.; Brauer, C.N.; Hidaka, K.; Löhmannsröben, H.-G.; Karthaus, O. Preparation of Patterned Zinc Oxide Films by Breath Figure Templating. Langmuir 2010, 26, 12173-12176. [CrossRef]

74. Hirai, Y.; Yabu, H.; Matsuo, Y.; Ijiro, K.; Shimomura, M. Biomimetic bi-functional silicon nanospike-array structures prepared by using self-organized honeycomb templates and reactive ion etching. J. Mater. Chem. 2010, 20, 10804-10808. [CrossRef]

75. Pisco, M.; Galeotti, F.; Quero, G.; Iadicicco, A.; Giordano, M.; Cusano, A. Miniaturized Sensing Probes Based on Metallic Dielectric Crystals Self-Assembled on Optical Fiber Tips. ACS Photonics 2014, 1, 917-927. [CrossRef]

76. Koo, K.; Ahn, H.; Kim, S.-W.; Ryu, D.Y.; Russell, T.P. Directed self-assembly of block copolymers in the extreme: Guiding microdomains from the small to the large. Soft Matter 2013, 9, 9059-9071. [CrossRef]

77. Evangelio, L.; Fernández-Regúlez, M.; Borrisé, X.; Lorenzoni, M.; Fraxedas, J.; Pérez-Murano, F. Creation of guiding patterns for directed self-assembly of block copolymers by resistless direct e-beam exposure. J. Micro/Nanolithogr. MEMS MOEMS 2015, 14, 033511. [CrossRef]

78. Liu, C.-C.; Franke, E.; Mignot, Y.; Xie, R.; Yeung, C.W.; Zhang, J.; Chi, C.; Zhang, C.; Farrell, R.; Lai, K.; et al. Directed self-assembly of block copolymers for 7 nanometre FinFET technology and beyond. Nat. Electron. 2018, 1, 562-569. [CrossRef]

79. Tsai, H.; Guillorn, M.; Doerk, G.; Cheng, J.; Sanders, D.; Lai, K.; Liu, C.; Colburn, M. Directed self-assembly for ever-smaller printed circuits. Spie Newsroom 2013. [CrossRef]

80. Choi, H.K.; Chang, J.-B.; Hannon, A.F.; Yang, J.K.W.; Berggren, K.K.; Alexander-Katz, A.; Ross, C.A. Nanoscale spirals by directed self-assembly. Nano Futures 2017, 1, 015001. [CrossRef]

81. Grzelczak, M.; Vermant, J.; Furst, E.M.; Liz-Marzán, L.M. Directed Self-Assembly of Nanoparticles. ACS Nano 2010, 4, $3591-3605$. [CrossRef]

82. Cara, E.; Ferrarese Lupi, F.; Fretto, M.; De Leo, N.; Tortello, M.; Gonnelli, R.; Sparnacci, K.; Boarino, L. Directed Self-Assembly of Polystyrene Nanospheres by Direct Laser-Writing Lithography. Nanomaterials 2020, 10, 280. [CrossRef]

83. Yap, F.L.; Thoniyot, P.; Krishnan, S.; Krishnamoorthy, S. Nanoparticle Cluster Arrays for High-Performance SERS through Directed Self-Assembly on Flat Substrates and on Optical Fibers. ACS Nano 2012, 6, 2056-2070. [CrossRef]

84. Colusso, E.; De Ferrari, F.; Minzioni, P.; Martucci, A.; Wang, Y.; Omenetto, F.G. Engineering optical defects in biopolymer photonic lattices. J. Mater. Chem. C 2018, 6, 966-971. [CrossRef]

85. Rogers, J.A.; Lee, H.H.; Patterning with Block Copolymers. Unconventional Nanopatterning Techniques and Applications; John Wiley \& Sons: Hoboken, NJ, USA, 2008; pp. 233-289.

86. Baruth, A.; Seo, M.; Lin, C.H.; Walster, K.; Shankar, A.; Hillmyer, M.A.; Leighton, C. Optimization of Long-Range Order in Solvent Vapor Annealed Poly(styrene)-block-poly(lactide) Thin Films for Nanolithography. ACS Appl. Mater. Interf. 2014, 6, 13770-13781. [CrossRef]

87. Wei, X.; Zhuang, X.; Hong, S.-C.; Goto, T.; Shen, Y.R. Sum-Frequency Vibrational Spectroscopic Study of a Rubbed Polymer Surface. Phys. Rev. Lett. 1999, 82, 4256-4259. [CrossRef]

88. Amit, Y.; Faust, A.; Lieberman, I.; Yedidya, L.; Banin, U. Semiconductor nanorod layers aligned through mechanical rubbing. Phys. Status Solid. A 2012, 209, 235-242. [CrossRef]

89. Wei, Y.; Xu, Y.; Wang, Q.; Wang, J.; Lu, H.; Zhu, J. CsPbBr3 nanowire polarized light-emitting diodes through mechanical rubbing. Chem. Commun. 2020, 56, 5413-5416. [CrossRef]

90. Heil, H.; Finnberg, T.; Malm, N.v.; Schmechel, R.; Seggern, H.v. The influence of mechanical rubbing on the field-effect mobility in polyhexylthiophene. J. Appl. Phys. 2003, 93, 1636-1641. [CrossRef]

91. Park, C.; Lee, T.; Xia, Y.; Shin, T.J.; Myoung, J.; Jeong, U. Quick, Large-Area Assembly of a Single-Crystal Monolayer of Spherical Particles by Unidirectional Rubbing. Adv. Mater. 2014, 26, 4633-4638. [CrossRef]

92. Meng, X.; Qiu, D. Gas-Flow-Induced Reorientation to Centimeter-Sized Two-Dimensional Colloidal Single Crystal of Polystyrene Particle. Langmuir 2014, 30, 3019-3023. [CrossRef]

93. Ramananarivo, S.; Ducrot, E.; Palacci, J. Activity-controlled annealing of colloidal monolayers. Nat. Commun. 2019, 10, 3380. [CrossRef]

94. Toolan, D.T.W.; Fujii, S.; Ebbens, S.J.; Nakamura, Y.; Howse, J.R. On the mechanisms of colloidal self-assembly during spin-coating. Soft Matter 2014, 10, 8804-8812. [CrossRef]

95. Lotito, V.; Zambelli, T. Self-assembly and nanosphere lithography for large-area plasmonic patterns on graphene. J. Colloid Interf. Sci. 2015, 447, 202-210. [CrossRef]

96. Escalé, P.; Save, M.; Lapp, A.; Rubatat, L.; Billon, L. Hierarchical structures based on self-assembled diblock copolymers within honeycomb micro-structured porous films. Soft Matter 2010, 6, 3202-3210. [CrossRef]

97. Sulka, G.D.; Kapusta-Kołodziej, J.; Brzózka, A.; Jaskuła, M. Fabrication of nanoporous TiO2 by electrochemical anodization. Electrochim. Acta 2010, 55, 4359-4367. [CrossRef]

98. Bigioni, T.P.; Lin, X.-M.; Nguyen, T.T.; Corwin, E.I.; Witten, T.A.; Jaeger, H.M. Kinetically driven self assembly of highly ordered nanoparticle monolayers. Nat. Mater. 2006, 5, 265-270. [CrossRef] 
99. Rengarajan, R.; Mittleman, D.; Rich, C.; Colvin, V. Effect of disorder on the optical properties of colloidal crystals. Phys. Rev. E 2005, 71, 016615. [CrossRef]

100. Schneider, C.A.; Rasband, W.S.; Eliceiri, K.W. NIH Image to ImageJ: 25 years of image analysis. Nat. Methods 2012, 9, 671-675. [CrossRef]

101. Bumstead, M.; Liang, K.; Hanta, G.; Hui, L.S.; Turak, A. disLocate: Tools to rapidly quantify local intermolecular structure to assess two-dimensional order in self-assembled systems. Sci. Rep. 2018, 8, 1554. [CrossRef]

102. Chieffi, G.; Di Girolamo, R.; Aronne, A.; Pernice, P.; Fanelli, E.; Lazzari, M.; De Rosa, C.; Auriemma, F. Rapid-flux-solventatmosphere method for tailoring the morphology of titania substrates over a large area via direct self-assembly of block copolymers. RSC Adv. 2014, 4, 16721-16725. [CrossRef]

103. Park, M.S.; Kim, J.K. Breath Figure Patterns Prepared by Spin Coating in a Dry Environment. Langmuir 2004, $20,5347-5352$. [CrossRef] [PubMed]

104. Prevo, B.G.; Velev, O.D. Controlled, Rapid Deposition of Structured Coatings from Micro- and Nanoparticle Suspensions. Langmuir 2004, 20, 2099-2107. [CrossRef] [PubMed]

105. Smith, N.L.; Coukouma, A.; Dubnik, S.; Asher, S.A. Debye ring diffraction elucidation of 2D photonic crystal self-assembly and ordering at the air-water interface. Phys. Chem. Chem. Phys. 2017, 19, 31813-31822. [CrossRef]

106. Kim, M.H.; Im, S.H.; Park, O.O. Rapid Fabrication of Two- and Three-Dimensional Colloidal Crystal Films via Confined Convective Assembly. Adv. Funct. Mater. 2005, 15, 1329-1335. [CrossRef]

107. Dai, Z.; Li, Y.; Duan, G.; Jia, L.; Cai, W. Phase Diagram, Design of Monolayer Binary Colloidal Crystals, and Their Fabrication Based on Ethanol-Assisted Self-Assembly at the Air/Water Interface. ACS Nano 2012, 6, 6706-6716. [CrossRef]

108. Diba, F.S.; Boden, A.; Thissen, H.; Bhave, M.; Kingshott, P.; Wang, P.-Y. Binary colloidal crystals (BCCs): Interactions, fabrication, and applications. Adv. Colloid Interf. Sci. 2018, 261, 102-127. [CrossRef]

109. Vogel, N.; de Viguerie, L.; Jonas, U.; Weiss, C.K.; Landfester, K. Wafer-Scale Fabrication of Ordered Binary Colloidal Monolayers with Adjustable Stoichiometries. Adv. Funct. Mater. 2011, 21, 3064-3073. [CrossRef]

110. Paik, T.; Diroll, B.T.; Kagan, C.R.; Murray, C.B. Binary and Ternary Superlattices Self-Assembled from Colloidal Nanodisks and Nanorods. J. A. Chem. Soc. 2015, 137, 6662-6669. [CrossRef] 